\title{
On the stability of periodically time-dependent quantum systems
}

\author{
P. Duclos ${ }^{1}$, E. Soccorsi ${ }^{1}$, P. Šťovíček ${ }^{2}$, M. Vittot ${ }^{1}$
}

October 24, 2018

\begin{abstract}
${ }^{1}$ Centre de Physique théorique de Marseille UMR 6207 - Unité Mixte de Recherche du CNRS et des Universités Aix-Marseille I, Aix-Marseille II et de l' Université du Sud Toulon-Var - Laboratoire affilié à la FRUMAM

${ }^{2}$ Department of Mathematics, Faculty of Nuclear Science, Czech Technical University, Trojanova 13, 12000 Prague, Czech Republic
\end{abstract}

\begin{abstract}
The main motivation of this article is to derive sufficient conditions for dynamical stability of periodically driven quantum systems described by a Hamiltonian $H(t)$, i.e., conditions under which it holds $\sup _{t \in \mathbb{R}}\left|\left\langle\psi_{t}, H(t) \psi_{t}\right\rangle\right|<\infty$ where $\psi_{t}$ denotes a trajectory at time $t$ of the quantum system under consideration. We start from an analysis of the domain of the quasi-energy operator. Next we show, under certain assumptions, that if the spectrum of the monodromy operator $U(T, 0)$ is pure point then there exists a dense subspace of initial conditions for which the mean value of energy is uniformly bounded in the course of time. Further we show that if the propagator admits a differentiable Floquet decomposition then $\left\|H(t) \psi_{t}\right\|$ is bounded in time for any initial condition $\psi_{0}$, and one employs the quantum KAM algorithm to prove the existence of this type of decomposition for a fairly large class of $H(t)$. In addition, we derive bounds uniform in time on transition probabilities between different energy levels, and we also propose an extension of this approach to the case of a higher order of differentiability of the Floquet decomposition. The procedure is demonstrated on a solvable example of the periodically time-dependent harmonic oscillator.
\end{abstract}

\section{Introduction}

We discuss several topics related to the dynamical properties of periodically timedependent quantum systems. Such a system is described by a Hamiltonian $H(t)$ in a Hilbert space $\mathscr{H}$ depending on $t$ periodically with a period $T$, and we suppose that the propagator $U(t, s)$ associated to the Hamiltonian $H(t)$ exists. 
We start our exposition from an analysis of the domain of the Floquet Hamiltonian (the quasi-energy operator). The quasi-energy operator is a basic tool in the theory of time-dependent quantum systems and is closely related to the monodromy operator $U(T, 0)[16,26$. This is a common belief that the dynamical properties are essentially determined by the spectral properties of $U(T, 0)$. It is shown in [13] that $\psi$ belongs to $\mathscr{H}^{p p}(U(T, 0))$ (the subspace in $\mathscr{H}$ corresponding to the pure point spectrum of $U(T, 0)$ ) if and only if the trajectory $\left\{\psi_{t} ; t \geq 0\right\}$ is precompact (where $\psi_{t}=U(t, 0) \psi$ ). Under the assumptions that $H(0)$ is positive, discrete and unbounded, and that the perturbation $H(t)-H(0)$ is uniformly bounded, it is observed in [20] that if the mean value of energy, $\left\langle\psi_{t}, H(t) \psi_{t}\right\rangle$, is bounded then the corresponding trajectory $\left\{\psi_{t} ; t \geq 0\right\}$ is precompact. Jointly this implies that if the mean value of energy is bounded for any initial condition then $U(T, 0)$ has a pure point spectrum. To our knowledge, the inverse implication is not clarified yet. In the present paper we show, under certain assumptions, that if the spectrum of $U(T, 0)$ is pure point then there exists, in $\mathscr{H}$, a dense subspace of initial conditions for which the mean value of energy is bounded. However it has been shown very recently in 21 that there exist situations when some trajectories may lead to unbounded energy in spite of pure pointness of $U(T, 0)$.

There is no doubt that the knowledge of evolution of the mean value of energy in the case of time-dependent systems is important from the physical point of view. This is also our basic topic in this paper. More precisely, instead of treating directly the mean value of energy we consider the quantity $\left\|H(t) \psi_{t}\right\|$. Naturally, this type of problems attracted attention in the past though the results are less numerous than one might expect. Let us mention some of them that motivated us though in no way we attempt to provide an exhaustive list.

Assuming a growing gap structure of the spectrum of $H(0)$ it is shown in 19 with the aid of adiabatic methods that $\left\langle\psi_{t}, H(t) \psi_{t}\right\rangle=O\left(t^{\delta}\right)$ where $\delta>0$ is inversely proportional to the order of differentiability of $H(t)$. An upper bound of this type is also derived in [17] under rather mild assumptions on the gap structure of the spectrum and without differentiability of $H(t)$. On the other hand, the latter result is directly applicable only provided the perturbation is in certain sense small when compared to $H(0)$. For example, in the case of simple spectrum the operator $H(0)^{q}(H(t)-H(0))$ is required to be Hilbert-Schmidt for some $q \geq 1 / 2$. Some extensions and applications can be also found in [3]. These estimates on the growth of energy were derived without assuming the periodicity. Let us also mention [10] where bounds on the energy growth are derived in the case of shrinking gaps in the spectrum.

A stronger result is known for periodically time-dependent systems [1]. It suggests that for a large class of periodic systems one can expect uniform boundedness of the mean value of energy for any initial condition $\psi \in \operatorname{Dom} H(0)$. Further, in [25] the energy is shown to be uniformly bounded in time in the particular case when the harmonic oscillator is driven by quasi-periodically time dependent Gaussian potentials for suitable non resonant frequencies and a small enough coupling constant. It is proposed in [7] to call this property dynamical stability. We adopt this terminology in the current paper.

Though the ideas concerning the dynamical stability are developed in [1] on a 
particular example of the driven ring it is indicated there that they are valid also under more general settings. The proof is based on two observations. First, if the propagator admits a differentiable Floquet decomposition in the sense that it can be written in the form $U(t)=U_{F}(t) \exp \left(-\mathrm{i} t H_{F}\right)$ where $H_{F}$ is self-adjoint and $U_{F}(t)$ is a periodic and strongly differentiable family of unitary operators then the system is dynamically stable. According to the second observation one can use the quantum KAM (Kolmogorov-Arnold-Moser) algorithm to show that the propagator actually admits this type of decomposition in the case when $H(0)$ is a semi-bounded discrete operator obeying a gap condition, and provided the frequency is non-resonant and the time-dependent perturbation is sufficiently small. In particular, the result in [1] is based on a formulation of the quantum KAM theorem presented in 12 .

In the current paper we wish to further develop the basic ideas from [1] and particularly to work out the proofs in full detail when considering applications of these ideas to more general systems. In addition we derive uniform bounds on transition probabilities between different energy levels. Moreover, we propose an extension to the case when the Floquet decomposition is $p$ times continuously differentiable in the strong sense. Restricting the perturbation $V(t)=H(t)-H(0)$ to a certain class of operator-valued functions by requiring the multiple commutators with $H(0)$ to be bounded up to some order one can show that $\left\|H(t)^{p} \psi_{t}\right\|$ is bounded in time. Furthermore, the basic procedure is demonstrated on the solvable example of the periodically time-dependent harmonic oscillator. For the purposes of this example we collect in the Appendix some useful formulas for the propagator. Finally we combine the procedure based on the differentiability of the Floquet decomposition with an improved version of the quantum KAM theorem that was presented in [11].

\section{The Floquet Hamiltonian}

Let us make more precise the assumptions on the Hamiltonian. Let $\{H(t) ; t \in \mathbb{R}\}$ be a family of self-adjoint operators such that the domain $\operatorname{Dom}(H(t))$ does not depend on time. Further we assume that the propagator $U(t, s)$ associated to $H(t)$ exists. This means that $U(t, s)$ is a function with values in $\mathscr{B}(\mathscr{H})$ which is strongly continuous jointly in $t$ and $s, U(t, t)=\mathbb{I}$, the domain $\operatorname{Dom}(H(0))$ is invariant under the action of $U(t, s)$ for all $t, s$, and

$$
\forall \psi \in \operatorname{Dom}(H(0)), \text { i } \partial_{t} U(t, s) \psi=H(t) U(t, s) \psi .
$$

Then the propagator is unique, unitary and satisfies the Chapman-Kolmogorov equation: $U(t, r) U(r, s)=U(t, s)$ for all $t, r, s \in \mathbb{R}$.

Let us recall that usually one imposes a standard sufficient condition that guarantees the existence of the evolution operator. Namely, if the mapping

$$
t, s \mapsto \frac{1}{t-s}\left((H(t)+\mathrm{i})(H(s)+\mathrm{i})^{-1}-\mathbb{I}\right)
$$

can be extended for $t=s$ to a strongly continuous mapping $\mathbb{R}^{2} \rightarrow \mathscr{B}(\mathscr{H})$ then the propagator exists [22]. For more general sufficient conditions one can consult the 
monographs [24] and [18]. But as already stated, we assume directly the existence of the propagator without bothering about particular hypotheses that guarantee it.

Since the Hamiltonian $H(t)$ is assumed to be $T$-periodic the same is true for the propagator. This means that

$$
\forall t, s, U(t+T, s+T)=U(t, s) .
$$

Notice also that by the closed graph theorem the operator $H(t)(H(0)+\mathrm{i})^{-1}$ is bounded. In addition, in this section we impose the following two assumptions:

$$
\mathbb{R} \ni t \mapsto\left\|H(t)(H(0)+\mathrm{i})^{-1}\right\| \text { is locally bounded, }
$$

$\forall \psi \in \operatorname{Dom}(H(0)), \mathbb{R} \ni t \mapsto\|H(0) U(t, 0) \psi\|$ is locally square integrable.

In fact, hypothesis (2) means that $H(t)(H(0)+\mathrm{i})^{-1}$ is bounded uniformly in $t$ since we are considering the periodic case.

An important tool when investigating time dependent quantum systems is the Floquet Hamiltonian (also called the quasi-energy operator) [16, 26]. It acts in the Hilbert space

$$
\mathscr{K}=L^{2}([0, T], \mathscr{H}, \mathrm{d} t) \equiv L^{2}([0, T], \mathrm{d} t) \otimes \mathscr{H} .
$$

If convenient we shall regard the elements of $\mathscr{K}$ as $T$-periodic vector-valued functions on $\mathbb{R}$ with values in $\mathscr{H}$. A unique Floquet Hamiltonian is associated to any strongly continuous propagator via the Stone theorem according to the prescription

$$
\forall f \in \mathscr{K}, \forall \sigma \in \mathbb{R} \text {, for a.a. } t \in \mathbb{R},\left(e^{-\mathrm{i} \sigma K} f\right)(t)=U(t, t-\sigma) f(t-\sigma) .
$$

Hence $f$ belongs to $\operatorname{Dom}(K)$ if and only if the derivative i $\left.\partial_{\sigma} U(t, t-\sigma) f(t-\sigma)\right|_{\sigma=0}$ exists in $\mathscr{K}$. Morally the Floquet Hamiltonian can be regarded as $-\mathrm{i} \partial_{t}+H(t)$ but in general this formal expression should be interpreted in a weak sense. The following remarks aim to provide some details about the definition of $K$.

In the particular case when the Hamiltonian does not depend on time and equals $H_{0}$ for all $t$ it holds $U(t, t-\sigma)=\exp \left(-\mathrm{i} \sigma H_{0}\right)$ and one easily finds from (4) that the associated Floquet Hamiltonian $K_{0}$ is nothing but the closure of the operator $-\mathrm{i} \partial_{t} \otimes 1+1 \otimes H_{0}$ defined on the algebraic tensor product $\operatorname{Dom}\left(\mathrm{i} \partial_{t}\right) \otimes \operatorname{Dom}\left(H_{0}\right)$. Here and everywhere in what follows the time derivative is automatically considered with the periodic boundary conditions. This is to say that the orthonormal basis $\left\{T^{-1 / 2} \exp (2 \pi \mathrm{i} k t / T) ; k \in \mathbb{Z}\right\}$ in $L^{2}([0, T], \mathrm{d} t)$ is formed by eigenfunctions of $\mathrm{i} \partial_{t}$.

Let us denote by $C_{T}^{\infty}(\mathbb{R})$ the space of $T$-periodic smooth functions on $\mathbb{R}$ and let

$$
C_{T}^{\infty}(\mathbb{R}) \otimes \operatorname{Dom}(H(0))=\operatorname{span}\left\{\eta(t) \psi ; \eta \in C_{T}^{\infty}(\mathbb{R}), \psi \in \operatorname{Dom}(H(0))\right\} \subset \mathscr{K}
$$

be the algebraic tensor product. It is straightforward to see that $C_{T}^{\infty}(\mathbb{R}) \otimes \operatorname{Dom}(H(0)) \subset$ $\operatorname{Dom}(K)$ and

$$
K(\eta \otimes \psi)(t)=-\mathrm{i} \eta^{\prime}(t) \psi+\eta(t) H(t) \psi,
$$

for every $\eta \in C_{T}^{\infty}(\mathbb{R})$ and $\psi \in \operatorname{Dom}(H(0))$. Set

$$
K^{0}=\left.K\right|_{C_{T}^{\infty}(\mathbb{R}) \otimes \operatorname{Dom}(H(0))} .
$$


It follows that $K^{0}$ is a symmetric operator, $K^{0} \subset K \subset\left(K^{0}\right)^{*}$.

Let $K^{1}$ be another operator acting in $\mathscr{K}$ and defined by the prescription: $f \in$ $\operatorname{Dom}\left(K^{1}\right)$ if and only if, for every $\psi \in \operatorname{Dom}(H(0))$, the function $t \mapsto\langle\psi, f(t)\rangle_{\mathscr{H}}$ is absolutely continuous and there exists $g \in \mathscr{K}$ such that

$$
\forall \psi \in \operatorname{Dom}(H(0)), \quad-\mathrm{i} \partial_{t}\langle\psi, f(t)\rangle_{\mathscr{H}}+\langle H(t) \psi, f(t)\rangle_{\mathscr{H}}=\langle\psi, g(t)\rangle_{\mathscr{H}},
$$

(the last equality is valid, of course, almost everywhere on $\mathbb{R}$ ). In that case $g$ is unique and we set $K^{1} f=g$.

From the definition it is obvious that $K^{0} \subset K^{1}$. Hence $K$ and $K^{1}$ coincide on $C_{T}^{\infty}(\mathbb{R}) \otimes \operatorname{Dom}(H(0))$. We shall show that $K$ and $K^{1}$ are actually equal. Let us make a remark on the notation used below and everywhere in the remainder of the paper: the natural numbers $\mathbb{N}$ start from 1 while $\mathbb{Z}_{+}$stands for non-negative integers.

Lemma 1. For all $\psi \in \mathscr{H}$ and $f \in \operatorname{Dom}\left(K^{1}\right)$, the function $\langle U(t, 0) \psi, f(t)\rangle_{\mathscr{H}}$ is absolutely continuous and it holds true that

$$
-\mathrm{i} \partial_{t}\langle U(t, 0) \psi, f(t)\rangle_{\mathscr{H}}=\langle U(t, 0) \psi, g(t)\rangle_{\mathscr{H}} \text { for a.e. } t \in \mathbb{R},
$$

where $g=K^{1} f$.

Proof. Let us first suppose that $\psi \in \operatorname{Dom}(H(0))$. Let $P$ be the projector-valued measure for $H(0)$ and set $P_{n}=P([-n, n]), n \in \mathbb{N}$. Then $P_{n} \rightarrow \mathbb{I}$ strongly as $n \rightarrow \infty$ and therefore the following limit is true in the space of distributions $\mathscr{D}^{\prime}(\mathbb{R})$ (actually in $\left.L_{\text {loc }}^{1}(\mathbb{R})\right)$ :

$$
\lim _{n \rightarrow \infty}\left\langle U(t, 0) \psi, P_{n} f(t)\right\rangle_{\mathscr{H}}=\langle U(t, 0) \psi, f(t)\rangle_{\mathscr{H}} .
$$

We shall compute the time derivative of $\langle U(t, 0) \psi, f(t)\rangle_{\mathscr{H}}$ in the sense of distributions when making use of the fact that $-\mathrm{i} \partial_{t}$ is continuous on $\mathscr{D}^{\prime}(\mathbb{R})$. Choose an orthonormal basis in $\mathscr{H}$ called $\left\{\varphi_{k}\right\}$. The series

$$
\left\langle U(t, 0) \psi, P_{n} f(t)\right\rangle_{\mathscr{H}}=\sum_{k}\left\langle U(t, 0) \psi, \varphi_{k}\right\rangle_{\mathscr{H}}\left\langle\varphi_{k}, P_{n} f(t)\right\rangle_{\mathscr{H}}
$$

converges in $\mathscr{D}^{\prime}(\mathbb{R})$ (actually in $L_{\text {loc }}^{1}(\mathbb{R})$ ) since it converges absolutely and is majorized by $\|\psi\|_{\mathscr{H}}\|f(t)\|_{\mathscr{H}}$, a locally integrable function. Then, in the sense of distributions,

$$
\begin{aligned}
-\mathrm{i} \partial_{t}\left\langle U(t, 0) \psi, P_{n} f(t)\right\rangle_{\mathscr{H}}=\sum_{k}\left(\left\langle H(t) U(t, 0) \psi, \varphi_{k}\right\rangle_{\mathscr{H}}\left\langle\varphi_{k}, P_{n} f(t)\right\rangle_{\mathscr{H}}\right. \\
\left.-\left\langle U(t, 0) \psi, \varphi_{k}\right\rangle_{\mathscr{H}}\left\langle H(t) P_{n} \varphi_{k}, f(t)\right\rangle_{\mathscr{H}}+\left\langle U(t, 0) \psi, \varphi_{k}\right\rangle_{\mathscr{H}}\left\langle\varphi_{k}, P_{n} g(t)\right\rangle_{\mathscr{H}}\right) .
\end{aligned}
$$

Here we have used the definition of $K^{1}$ (note that $P_{n} \varphi_{k} \in \operatorname{Dom}(H(0))$ ).

The RHS in (77) splits into three sums each of them can be summed in $\mathscr{D}^{\prime}(\mathbb{R})$. To see it let us note that with the aid of the Schwarz inequality and the Parseval equality one can estimate

$$
\sum_{k}\left|\left\langle H(t) U(t, 0) \psi, \varphi_{k}\right\rangle_{\mathscr{H}}\left\langle\varphi_{k}, P_{n} f(t)\right\rangle_{\mathscr{H}}\right| \leq\|H(t) U(t, 0) \psi\|_{\mathscr{H}}\|f(t)\|_{\mathscr{H}} .
$$


Furthermore, $\|f(t)\|_{\mathscr{H}}$ is square integrable and

$$
\|H(t) U(t, 0) \psi\|_{\mathscr{H}} \leq\left\|H(t)(H(0)+\mathrm{i})^{-1}\right\|\|(H(0)+\mathrm{i}) U(t, 0) \psi\|_{\mathscr{H}}
$$

is locally square integrable due to (21) and (3). Hence the RHS of (8) is locally integrable. As far as the second sum is concerned let us note that

$$
G_{n}(t):=H(t) P_{n}=H(t)(H(0)+\mathrm{i})^{-1}(H(0)+\mathrm{i}) P_{n}
$$

is a bounded operator and even $\left\|G_{n}(t)\right\|$ is locally bounded according to hypothesis (2). Finally, the third sum does not cause any problem. Consequently, the RHS of (7) equals

$$
\left\langle H(t) U(t, 0) \psi, P_{n} f(t)\right\rangle_{\mathscr{H}}-\left\langle U(t, 0) \psi, G_{n}(t)^{*} f(t)\right\rangle_{\mathscr{H}}+\left\langle U(t, 0) \psi, P_{n} g(t)\right\rangle_{\mathscr{H}} .
$$

Thus $-\mathrm{i} \partial_{t}\langle U(t, 0) \psi, f(t)\rangle_{\mathscr{H}}$ is equal to the limit of (9) as $n \rightarrow \infty$.

Since for every $\varphi \in \operatorname{Dom}(H(0))$ it holds $H(0) P_{n} \varphi \rightarrow H(0) \varphi$ and $U(t, 0) \psi \in$ $\operatorname{Dom}(H(0))$, in the second term in (9) we get

$$
\lim _{n \rightarrow \infty} G_{n}(t) U(t, 0) \psi=\lim _{n \rightarrow \infty} H(t)(H(0)+\mathrm{i})^{-1}(H(0)+\mathrm{i}) P_{n} U(t, 0) \psi=H(t) U(t, 0) \psi .
$$

The point-wise limits of the first and the third term in (9) are obvious. To justify the convergence in $\mathscr{D}^{\prime}(\mathbb{R})$ one can apply once more assumptions (2) and (3) to show that each term has a locally integrable majorant which is independent of $n$. Thus sending $n \rightarrow \infty$ one finds that equality (6) holds true in the sense of distributions. The RHS is a locally integrable function. By a standard result of the theory of distributions this implies that the function $\langle U(t, 0) \psi, f(t)\rangle_{\mathscr{H}}$ is absolutely continuous and that equality (6) holds true in the usual sense.

Finally let us show that the condition $\psi \in \operatorname{Dom}(H(0))$ from the beginning of the proof can be relaxed. Actually, if $h \in \mathscr{K}$ and $\psi_{k} \rightarrow \psi$ in $\mathscr{H}$ then $\left\langle U(t, 0) \psi_{k}, h(t)\right\rangle_{\mathscr{H}}$ is locally integrable and this sequence of functions converges to $\langle U(t, 0) \psi, h(t)\rangle_{\mathscr{H}}$ in the $L^{1}$ norm on every bounded interval and hence in the sense of distributions. For any $\psi \in \mathscr{H}$ one can choose a sequence $\psi_{k} \in \operatorname{Dom}(H(0))$ such that $\psi_{k} \rightarrow \psi$ and then send $k \rightarrow \infty$ in the equality

$$
-\mathrm{i} \partial_{t}\left\langle U(t, 0) \psi_{k}, f(t)\right\rangle_{\mathscr{H}}=\left\langle U(t, 0) \psi_{k}, g(t)\right\rangle_{\mathscr{H}} \text { in } \mathscr{D}^{\prime}(\mathbb{R}) .
$$

Since the function $\langle U(t, 0) \psi, g(t)\rangle_{\mathscr{H}}$ is locally integrable the function $\langle U(t, 0) \psi, f(t)\rangle_{\mathscr{H}}$ can be redefined on a measure zero set so that it is absolutely continuous and equality (6) holds true in the usual sense.

Lemma 2. $K^{1}$ is symmetric.

Proof. Suppose that $f \in \operatorname{Dom}\left(K^{1}\right), K^{1} f=g$ and $\psi \in \mathscr{H}$. According to Lemma 1 we have

$$
-\mathrm{i} \partial_{t}\left|\langle U(t, 0) \psi, f(t)\rangle_{\mathscr{H}}\right|^{2}=2 \mathrm{i} \operatorname{Im}\left(\langle f(t), U(t, 0) \psi\rangle_{\mathscr{H}}\langle U(t, 0) \psi, g(t)\rangle_{\mathscr{H}}\right)
$$


Let $\left\{\psi_{k}\right\}$ be an orthonormal basis in $\mathscr{H}$. Then, for almost all $s \in \mathbb{R}$ and all $k$,

$$
\begin{aligned}
\mid\left\langle\psi_{k}, U(s\right. & \left.+T, 0)^{-1} f(s+T)\right\rangle\left._{\mathscr{H}}\right|^{2}-\left|\left\langle\psi_{k}, U(s, 0)^{-1} f(s)\right\rangle_{\mathscr{H}}\right|^{2} \\
& =-2 \operatorname{Im} \int_{s}^{s+T}\left\langle U(t, 0)^{-1} f(t), \psi_{k}\right\rangle_{\mathscr{H}}\left\langle\psi_{k}, U(t, 0)^{-1} g(t)\right\rangle_{\mathscr{H}} \mathrm{d} t .
\end{aligned}
$$

Summing in $k$ one can commute the sum and the integral. Consequently, for almost all $s$,

$$
\|f(s+T)\|_{\mathscr{H}}^{2}-\|f(s)\|_{\mathscr{H}}^{2}=-2 \operatorname{Im} \int_{s}^{s+T}\langle f(t), g(t)\rangle_{\mathscr{H}} \mathrm{d} t=-2 \operatorname{Im}\left(\langle f, g\rangle_{\mathscr{K}}\right) .
$$

Since $\|f(t)\|_{\mathscr{H}}$ is periodic the LHS vanishes almost everywhere. We find that $\forall f \in$ $\operatorname{Dom}\left(K^{1}\right), \operatorname{Im}\left(\left\langle f, K^{1} f\right\rangle_{\mathscr{K}}\right)=0$. This shows that $K^{1}$ is symmetric.

Lemma 3. $\left(K^{0}\right)^{*}=K^{1}$. Consequently, $K^{1}$ is closed and $K^{0}$ is essentially self-adjoint. Proof. By definition, $f \in \operatorname{Dom}\left(\left(K^{0}\right)^{*}\right)$ if and only if there exists $g \in \mathscr{K}$ such that

$$
\forall h \in \operatorname{Dom}\left(K^{0}\right),\left\langle K^{0} h, f\right\rangle_{\mathscr{K}}=\langle h, g\rangle_{\mathscr{K}} .
$$

Moreover, in that case $g$ is unique and $\left(K^{0}\right)^{*} f=g$. Setting $h=\eta \otimes \psi$ we find that for all $\psi \in \operatorname{Dom}(H(0))$ it is true that

$$
\forall \eta \in C_{T}^{\infty}(\mathbb{R}), \quad \int_{0}^{T}\left(\mathrm{i} \eta^{\prime}(t)\langle\psi, f(t)\rangle_{\mathscr{H}}+\eta(t)\langle H(t) \psi, f(t)\rangle_{\mathscr{H}}\right) \mathrm{d} t=\int_{0}^{T} \eta(t)\langle\psi, g(t)\rangle_{\mathscr{H}} \mathrm{d} t .
$$

The last statement can be rewritten as equality (5) valid in the sense of distributions. Since the both functions $\langle H(t) \psi, f(t)\rangle_{\mathscr{H}}$ and $\langle\psi, g(t)\rangle_{\mathscr{H}}$ belong to $L_{\text {loc }}^{1}(\mathbb{R})$ (using again (2) in the former case) the standard results of the theory of distributions tell us that $\langle\psi, f(t)\rangle_{\mathscr{H}}$ is actually absolutely continuous and equality (5) holds true in the usual sense. Thus we conclude that $f \in \operatorname{Dom}\left(K^{1}\right)$ and $K^{1} f=g$. Hence $\left(K^{0}\right)^{*} \subset K^{1}$. Now it suffices to apply Lemma 2, Actually, the relations

$$
\left(K^{0}\right)^{*} \subset K^{1} \subset\left(K^{1}\right)^{*} \subset\left(K^{0}\right)^{* *}=\overline{K^{0}} \subset\left(K^{0}\right)^{*}
$$

imply that $K^{1}=\left(K^{0}\right)^{*}$ is closed and $\left(\overline{K^{0}}\right)^{*}=\left(K^{0}\right)^{*}=\overline{K^{0}}$.

Proposition 4. Assuming (2) and (3), it holds true that

$$
K=K^{1}=\overline{K^{0}}
$$

In particular, $C_{T}^{\infty}(\mathbb{R}) \otimes \operatorname{Dom}(H(0))$ is a core of $K$.

Proof. According to Lemma 2 and Lemma 3 it holds true that

$$
K^{0} \subset \overline{K^{0}} \subset K=K^{*} \subset\left(K^{0}\right)^{*}=\overline{K^{0}}
$$

and $K^{1}=\left(K^{0}\right)^{*}=\overline{K^{0}}$. The proposition follows immediately. 
Let us note that if a vector-valued function $f(t)$ from the domain of $K$ is even known to be continuously differentiable (in the strong sense) then necessarily $f(t) \in$ $\operatorname{Dom}(H(0))$ for all $t$. Under this additional assumption we actually have

$$
(K f)(t)=-\mathrm{i} \partial_{t} f(t)+H(t) f(t)=g(t) .
$$

In the general case, however, one should use the weaker form (5). The relation between $K$ and the formal expression $-\mathrm{i} \partial_{t}+H(t)$ can be also expressed as follows. Let $H=$ $\int^{\oplus} H(t) \mathrm{d} t$ be the self-adjoint operator in $\mathscr{K}$ with the domain formed by those $f \in \mathscr{K}$ satisfying $f(t) \in \operatorname{Dom}(H(0))$ for a.a. $t$ and $\int_{0}^{T}\|H(t) f(t)\|^{2} \mathrm{~d} t<\infty$, with $(H f)(t)=$ $H(t) f(t)$. Clearly,

$$
\operatorname{Dom}(K) \supset \operatorname{Dom}\left(-\mathrm{i} \partial_{t} \otimes 1\right) \cap \operatorname{Dom}(H) \supset C_{T}^{\infty}(\mathbb{R}) \otimes \operatorname{Dom}(H(0))
$$

and therefore, according to Proposition 4, $\operatorname{Dom}\left(-\mathrm{i} \partial_{t} \otimes 1\right) \cap \operatorname{Dom}(H)$ is a core of $K$. Hence

$$
K=\overline{-\mathrm{i} \partial_{t} \otimes 1+H}
$$

\section{Boundedness of energy for a dense set of initial conditions}

In this section we consider slightly more general periodically time-dependent Hamiltonians $H(t), t \in \mathbb{R}$, than those presented in the beginning of Section 2, at least among those which are bounded below. We suppose that the Hamiltonian $H(t)$ is associated to a closed, densely defined and positive sesquilinear form $q(t)$, with a domain independent of $t$ :

$$
\operatorname{Dom} q(t)=\operatorname{Dom} q(0), \forall t \in \mathbb{R} .
$$

Assuming that the spectrum of $U(T, 0)$ is pure point we wish to construct a rich set of initial conditions for which the mean value of energy is uniformly bounded in time. It turns out that this is possible if the eigenvectors of $U(T, 0)$ belong to the form domain $\operatorname{Dom} q(0)$.

The space $\operatorname{Dom} q(0)$ endowed with the scalar product $\langle u, v\rangle_{1}=\langle u, v\rangle_{\mathscr{H}}+q(0)(u, v)$ is a Hilbert space denoted by $\mathscr{H}_{1}$, and we recall that

$$
\langle H(t) u, v\rangle_{\mathscr{H}}=q(t)(u, v), \forall u \in \operatorname{Dom} H(t), \forall v \in \mathscr{H}_{1},
$$

where

$\operatorname{Dom} H(t)=\left\{u \in \mathscr{H}_{1} ; \exists C_{u} \geq 0\right.$ s.t. $\left.\forall v \in \mathscr{H}_{1},|q(t)(u, v)| \leq C_{u}\|v\|_{\mathscr{H}}\right\}$.

We call $\mathscr{H}_{-1}$ the dual space of $\mathscr{H}_{1}$, that is to say the vector space of continuous conjugate linear forms on $\mathscr{H}_{1}$. For any $u \in \mathscr{H}$, the functional $v \mapsto\langle v, u\rangle_{\mathscr{H}}$ belongs to $\mathscr{H}_{-1}$ since $\left|\langle v, u\rangle_{\mathscr{H}}\right| \leq\|u\|_{\mathscr{H}}\|v\|_{\mathscr{H}} \leq\|u\|_{\mathscr{H}}\|v\|_{1}$, and we can also regard $\mathscr{H}$ as a subspace of $\mathscr{H}_{-1}$ with

$$
\|u\|_{-1}=\sup _{v \in \mathscr{H}_{1}, v \neq 0} \frac{\left|\langle u, v\rangle_{\mathscr{H}}\right|}{\|v\|_{1}} \leq\|u\|_{\mathscr{H}} .
$$


Thus

$$
\mathscr{H}_{1} \subset \mathscr{H} \subset \mathscr{H}_{-1}
$$

where the symbol $\subset$ means a topological embedding. Actually, $H(t)$ can be extended into an operator mapping $\mathscr{H}_{1}$ into $\mathscr{H}_{-1}$ provided there exists a constant $C_{t} \geq 0$ such that

$$
\forall u \in \mathscr{H}_{1}, q(t)(u, u) \leq C_{t}\|u\|_{1}^{2} .
$$

Let us denote by $\langle\cdot, \cdot\rangle_{-1,1}$ the dual pairing between $\mathscr{H}_{-1}$ and $\mathscr{H}_{1}$. This pairing is conjugate linear in the first and linear in the second argument. In other words, the embedding $\mathscr{H} \subset \mathscr{H}_{-1}$ means that $\langle\psi, g\rangle_{-1,1}=\langle\psi, g\rangle_{\mathscr{H}}$ for all $\psi \in \mathscr{H}$ and $g \in \mathscr{H}_{1}$, and the mapping $H(t): \mathscr{H}_{1} \rightarrow \mathscr{H}_{-1}$ is defined so that $\langle H(t) u, v\rangle_{-1,1}=q(t)(u, v)$ for all $u, v \in \mathscr{H}_{1}$.

In the remainder of this section, we will refer to the propagator $U(t, 0)$ associated to the family of Hamiltonians $H(t), t \in \mathbb{R}$. Its existence is implied by the following result which can be found in [24, Theorem II.27] and that we reproduce below for the reader's convenience.

Theorem 5. We assume that $q(t)$ satisfies (11) and that there is a constant $C \geq 1$ such that the operator $H(t)$ satisfies, for all $t \in \mathbb{R}$ :

1. $C^{-1}(H(0)+1) \leq H(t) \leq C(H(0)+1)$.

2. The derivative $\frac{\mathrm{d}}{\mathrm{d} t} H(t)^{-1}$ exists in the norm sense and

$$
\left\|\sqrt{H(t)}\left(\frac{d}{d t} H(t)^{-1}\right) \sqrt{H(t)}\right\| \leq C .
$$

Then, for any $\psi_{0} \in \mathscr{H}_{1}$ there is a unique function $\mathbb{R} \ni t \mapsto \psi(t) \in \mathscr{H}_{1}$ such that:

1. $\psi$ is $\mathscr{H}_{1}$-weakly continuous, i.e., for all $g \in \mathscr{H}_{-1}, t \mapsto\langle g, \psi(t)\rangle_{-1,1}$ is a continuous function.

2. $\psi$ is a weak solution of the Schrödinger equation in the following sense:

$$
\forall g \in \mathscr{H}_{1}, \quad-\mathrm{i} \frac{\mathrm{d}}{\mathrm{d} t}\langle g, \psi(t)\rangle_{\mathscr{H}}+q(t)(g, \psi(t))=0 \text { and } \psi(0)=\psi_{0} .
$$

3. For all $s \in \mathbb{R}$ we have

$$
\lim _{t \rightarrow s}\left\|\frac{\psi(t)-\psi(s)}{t-s}+\mathrm{i} H(t) \psi(t)\right\|_{-1}=0 .
$$

4. $\|\psi(t)\|_{\mathscr{H}}=\left\|\psi_{0}\right\|_{\mathscr{H}}$ for all $t \in \mathbb{R}$ and $t \mapsto \psi(t)$ is continuous in the norm topology in $\mathscr{H}$.

The propagator $U:(s, t) \in \mathbb{R}^{2} \mapsto U(t, s)$ associated to the Hamiltonian $H(t)$ is defined by $U(t, s) \psi(s)=\psi(t)$. It is unitary and strongly continuous according to point 4 .

For the proof of the main result of this section, Proposition 7 , we need the following lemma. 
Lemma 6. Let $\psi \in \mathscr{H}_{1}$ be an eigenfunction of the Floquet operator $U(T, 0)$. Then the function

$$
F_{\psi}(t):=\|H(t) U(t, 0) \psi\|_{-1}
$$

is bounded in $\mathbb{R}$ :

$$
\left\|F_{\psi}\right\|_{\infty}:=\sup _{t \in \mathbb{R}} F_{\psi}(t)<+\infty .
$$

Proof. First, we notice that the function $t \mapsto\left|\langle H(t) U(t, 0) \psi, g\rangle_{-1,1}\right|$, with $g \in \mathscr{H}_{1}$, is periodic with the period $T$. This can be seen from the equality

$$
U(t+T, 0) \psi=U(t+T, T) U(T, 0) \psi=\lambda U(t, 0) \psi,
$$

and

$\left|\langle H(t+T) U(t+T, 0) \psi, g\rangle_{-1,1}\right|=|\lambda|\left|\langle H(t+T) U(t, 0) \psi, g\rangle_{-1,1}\right|=\left|\langle H(t) U(t, 0) \psi, g\rangle_{-1,1}\right|$

since $|\lambda|=1$ and $H(t+T)=H(t)$.

Moreover, the $\mathscr{H}_{-1}$-valued function $t \mapsto H(t) U(t, 0) \psi$ is weakly continuous on $\mathbb{R}$. Indeed, for any given real numbers $s$ and $t$, we derive from the following obvious decomposition, with $g \in \mathscr{H}_{1}$,

$$
\begin{aligned}
& \langle H(t) \psi(t), g\rangle_{-1,1}-\langle H(s) \psi(s), g\rangle_{-1,1} \\
& =\left\langle H(t) \psi(t)-\mathrm{i} \frac{\psi(t)-\psi(s)}{t-s}, g\right\rangle_{-1,1}-\left\langle H(s) \psi(s)-\mathrm{i} \frac{\psi(t)-\psi(s)}{t-s}, g\right\rangle_{-1,1},
\end{aligned}
$$

that

$$
\begin{aligned}
& \left|\langle H(t) \psi(t), g\rangle_{-1,1}-\langle H(s) \psi(s), g\rangle_{-1,1}\right| \\
& \leq\left\|H(t) \psi(t)-\mathrm{i} \frac{\psi(t)-\psi(s)}{t-s}\right\|_{-1}\|g\|_{1}+\left|q(s)(\psi(s), g)-\mathrm{i} \frac{\langle\psi(t), g\rangle_{\mathscr{H}}-\langle\psi(s), g\rangle_{\mathscr{H}}}{t-s}\right| .
\end{aligned}
$$

Applying respectively points 3 and 2 of Theorem 5 one finds that the both terms on the RHS of the preceding inequality tend to zero as $t$ tends to $s$.

This implies that for every $g \in \mathscr{H}_{1}$ the function $t \mapsto\left|\langle H(t) U(t, 0) \psi, g\rangle_{-1,1}\right|$ is bounded on $\mathbb{R}$ (since we just check that it is periodic). From the uniform boundedness principle it follows that

$$
F_{\psi}(t)=\sup _{g \in \mathscr{H}_{1},\|g\|_{1}=1}\left|\langle H(t) U(t, 0) \psi, g\rangle_{-1,1}\right|
$$

is bounded on $\mathbb{R}$ as well.

Proposition 7. Let us suppose that the Floquet operator $U(T, 0)$ has a pure point spectrum and admits a basis $\mathcal{B}$ formed by eigenfunctions belonging to $\mathscr{H}_{1}$. Then the energy of the quantum system, when starting from any initial state $\psi \in \operatorname{span} \mathcal{B}$, the set of finite linear combinations of vectors from $\mathcal{B}$, is bounded in the course of time:

$$
\sup _{t \in \mathbb{R}}\left|\langle H(t) U(t, 0) \psi, U(t, 0) \psi\rangle_{-1,1}\right|=\sup _{t \in \mathbb{R}}|q(t)(U(t, 0) \psi, U(t, 0) \psi)|<\infty .
$$


Proof. Recall that by our assumptions $H(t)^{-1}$ is a bounded operator on $\mathscr{H}$ (see Theorem 5). If $u \in \mathscr{H}_{1}, v \in \mathscr{H}$, then $H(t)^{-1} v \in \operatorname{Dom} H(t)$ and $q(t)\left(u, H(t)^{-1} v\right)=\langle u, v\rangle_{\mathscr{H}}$. Consequently,

$$
\left\langle H(t) u, H(t)^{-1} v\right\rangle_{-1,1}=q(t)\left(u, H(t)^{-1} v\right)=\langle u, v\rangle_{\mathscr{H}} .
$$

We can assume that the basis $\mathcal{B}$ is orthonormal. For any given $\psi$ in $\mathcal{B} \subset \mathscr{H}_{1}$, we first notice that $\|U(t, 0) \psi\|_{1}$ is bounded by $F_{\psi}(t)$ defined in Lemma 6 up to a multiplicative constant $C$. Indeed, for any $g \in \mathscr{H}$ we have

$$
\left|\langle U(t, 0) \psi, g\rangle_{\mathscr{H}}\right|=\left|\left\langle H(t) U(t, 0) \psi, H(t)^{-1} g\right\rangle_{-1,1}\right| \leq\|H(t) U(t, 0) \psi\|_{-1}\left\|H(t)^{-1} g\right\|_{1},
$$

with

$\left\|H(t)^{-1} g\right\|_{1}^{2}=\left\langle H(t)^{-1} g,(H(0)+1) H(t)^{-1} g\right\rangle_{\mathscr{C}} \leq C\left\langle H(t)^{-1} g, g\right\rangle_{\mathscr{H}} \leq C\left\|H(t)^{-1} g\right\|_{1}\|g\|_{-1}$, according to assumption 1 in Theorem 5. Thus $\left\|H(t)^{-1} g\right\|_{1} \leq C\|g\|_{-1}$ and (12) becomes

$$
\left|\langle g, U(t, 0) \psi\rangle_{-1,1}\right|=\left|\langle U(t, 0) \psi, g\rangle_{\mathscr{H}}\right| \leq C\|H(t) U(t, 0) \psi\|_{-1}\|g\|_{-1}=C F_{\psi}(t)\|g\|_{-1} .
$$

Furthermore, $\mathscr{H}$ being dense in $\mathscr{H}_{-1}$ in the norm topology, inequality (13) remains valid for any $g \in \mathscr{H}_{-1}$ implying

$$
\|U(t, 0) \psi\|_{1}=\sup _{g \in \mathscr{H}_{-1}, g \neq 0} \frac{\left|\langle g, U(t, 0) \psi\rangle_{-1,1}\right|}{\|g\|_{-1}} \leq C F_{\psi}(t) .
$$

To complete the proof we pick a function $\varphi$ in $\operatorname{span} \mathcal{B}, \varphi=\sum_{i=1}^{N} c_{i} \psi_{i}$, with $\psi_{i} \in \mathcal{B}$ and $c_{i} \in \mathbb{C}$ for $i=1,2, \ldots, N$. The energy function of the quantum system with the initial condition $\varphi$ decomposes as

$$
E_{\varphi}(t) \equiv\langle H(t) U(t, 0) \varphi, U(t, 0) \varphi\rangle_{-1,1}=\sum_{i, j=1}^{N} c_{i} \overline{c_{j}}\left\langle H(t) U(t, 0) \psi_{i}, U(t, 0) \psi_{j}\right\rangle_{-1,1} .
$$

Therefore,

$$
\begin{aligned}
\left|E_{\varphi}(t)\right| & \leq \sum_{i, j=1}^{N}\left|c_{i}\right|\left|c_{j}\right|\left\|H(t) U(t, 0) \psi_{i}\right\|_{-1}\left\|U(t, 0) \psi_{j}\right\|_{1} \\
& \leq C \sum_{i, j=1}^{N}\left|c_{i}\right|\left|c_{j}\right| F_{\psi_{i}}(t) F_{\psi_{j}}(t) \leq C \max _{1 \leq i \leq N} F_{\psi_{i}}(t)^{2}\left(\sum_{i=1}^{N}\left|c_{i}\right|\right)^{2},
\end{aligned}
$$

according to (14), so we finally obtain

$$
\left|E_{\varphi}(t)\right| \leq C N\|\varphi\|^{2} \max _{1 \leq i \leq N}\left\|F_{\psi_{i}}\right\|_{\infty}^{2}
$$

by combining the Cauchy-Schwarz inequality with Lemma 6 . 


\section{Bounds on energy and transition probabilities}

The only assumptions needed in this section are that the domain Dom $H(t)$ of a $T$ periodic family of self-adjoint operators is time-independent and that the propagator $U(t, s)$ associated to $H(t)$ exists in the usual sense, as recalled in the beginning of Section 2,

By the spectral theorem, the Floquet (monodromy) operator $U(T, 0)$ can be written in the form $U(T, 0)=\exp \left(-\mathrm{i} T H_{F}\right)$ where $H_{F}$ is a self-adjoint operator. Of course, the choice of $H_{F}$ is highly ambiguous. Let $U_{F}(t)$ be the family of unitary operators defined by the equality

$$
U(t, 0)=U_{F}(t) e^{-\mathrm{i} t H_{F}} .
$$

Then $U_{F}(0)=\mathbb{I}$ and from the periodicity of $U(t, s)$ (see (1)) it follows that $U_{F}(t)$ also depends on $t$ periodically. Relation (15) is known as the Floquet decomposition.

Definition 8. We shall say that a Floquet decomposition is $r$ times continuously differentiable in the strong sense for some $r \in \mathbb{N}$ if this is case for the family $U_{F}(t)$.

Furthermore, we shall say that a Floquet decomposition is relatively continuously differentiable in the strong sense if the family $U_{F}(t)\left(H_{F}+\mathrm{i}\right)^{-1}$ is continuously differentiable in the strong sense. Equivalently this means that for all $\psi \in \operatorname{Dom} H_{F}$ the vector-valued function $U_{F}(t) \psi$ is continuously differentiable.

Assume that the propagator $U(t, s)$ admits a Floquet decomposition which is relatively continuously differentiable in the strong sense. Set

$$
S_{F}(t)=\mathrm{i} U_{F}(t)^{-1} \partial_{t} U_{F}(t), \quad \operatorname{Dom} S_{F}(t)=\operatorname{Dom} H_{F} .
$$

By the uniform boundedness principle, $S_{F}(t)$ is $H_{F}$-bounded for all $t \in \mathbb{R}$. Using the periodicity of $U_{F}(t)$ and applying again the uniform boundedness principle one finds that $S_{F}(t)\left(H_{F}+\mathrm{i}\right)^{-1}$ is bounded uniformly in $t$. Moreover, $S_{F}(t)$ is a symmetric operator.

If the Floquet decomposition is even continuously differentiable in the strong sense then $S_{F}(t)$ will be naturally supposed to be defined on the entire space $\mathscr{H}$. Referring again to the uniform boundedness principle, in this case we have $S_{F}(t) \in \mathscr{B}(\mathscr{H})$. Using the periodicity of $U_{F}(t)$ and applying the uniform boundedness principle once more one finds that $S_{F}(t)$ is bounded uniformly in $t$. Hence $S_{F}:=\int^{\oplus} S_{F}(t) \mathrm{d} t$ is a bounded operator in $\mathscr{K}$ whose norm equals

$$
\left\|S_{F}\right\|=\sup _{t \in \mathbb{R}}\left\|S_{F}(t)\right\|
$$

Moreover, $S_{F}(t)$ is a Hermitian operator.

Lemma 9. Assume that a Floquet decomposition (15) is relatively continuously differentiable in the strong sense and that the relative bound of $S_{F}(t)$ with respect to $H_{F}$ is less than one for all $t$. Then

$$
\forall t \in \mathbb{R}, H(t)=U_{F}(t)\left(H_{F}+S_{F}(t)\right) U_{F}(t)^{-1} .
$$


In particular,

$$
H(0)=H_{F}+S_{F}(0)
$$

Furthermore,

$$
\operatorname{Dom}\left(H_{F}\right)=\operatorname{Dom}(H(0))
$$

and this domain is $U_{F}(t)$ invariant.

Proof. By the assumptions and the Kato-Rellich theorem (see, for example, [22]),

$$
\widetilde{H}(t):=U_{F}(t)\left(H_{F}+S_{F}(t)\right) U_{F}(t)^{-1}, \quad \operatorname{Dom} \widetilde{H}(t)=U_{F}(t)\left(\operatorname{Dom} H_{F}\right),
$$

is a $T$-periodic family of self-adjoint operators. From (15) it follows that

$$
U(t, s)=U_{F}(t) e^{-\mathrm{i}(t-s) H_{F}} U_{F}(s)^{-1} .
$$

From this relation it is obvious that

$$
U(t, s)(\operatorname{Dom} \widetilde{H}(s))=\operatorname{Dom} \widetilde{H}(t) .
$$

Suppose that $\varphi \in U_{F}(s)\left(\operatorname{Dom} H_{F}\right)$ and thus $\varphi=U_{F}(s) \psi$ for some $\psi \in \operatorname{Dom} H_{F}$. A straightforward computation yields

$\mathrm{i} \partial_{t} U(t, s) \varphi=\mathrm{i} \partial_{t}\left(U_{F}(t) e^{-\mathrm{i}(t-s) H_{F}} \psi\right)=U_{F}(t)\left(H_{F}+S_{F}(t)\right) e^{-\mathrm{i}(t-s) H_{F}} \psi=\widetilde{H}(t) U(t, s) \varphi$.

Hence $U(t, s)$ is a propagator associated to the family $\widetilde{H}(t)$.

Using the property of self-adjointness one can easily see that the uniqueness of the relation between a Hamiltonian and a propagator applies also in the following direction: if two (in general time-dependent) Hamiltonians generate the same propagator then they are equal. In our case this means that $\widetilde{H}(t)=H(t)$ for all $t$, i.e., equality (17) holds true. Consequently, $U_{F}(t)\left(\operatorname{Dom} H_{F}\right)=\operatorname{Dom} H(t)=\operatorname{Dom} H(0)$ and setting $t=0$ we have $\operatorname{Dom} H_{F}=\operatorname{Dom} H(0)$.

Next we shall show that the relative continuous differentiability of $U_{F}(t)$ implies the dynamical stability.

Proposition 10. Under the same assumptions as in Lemma 9, the energy of the system described by the Hamiltonian $H(t)$ is uniformly bounded for any initial condition. More precisely,

$$
\forall \psi \in \operatorname{Dom}(H(0)), \sup _{t \in \mathbb{R}}\|H(t) U(t, 0) \psi\| \leq C_{\psi}
$$

where

$$
C_{\psi}=\left\|H_{F} \psi\right\|+\sup _{t}\left\|S_{F}(t)\left(H_{F}+\mathrm{i}\right)^{-1}\right\|\left\|\left(H_{F}+\mathrm{i}\right) \psi\right\| .
$$

Proof. From equalities (15) and (17) it follows that

$$
\|H(t) U(t, 0) \psi\|=\left\|\left(H_{F}+S_{F}(t)\right) e^{-\mathrm{i} t H_{F}} \psi\right\| \leq C_{\psi} .
$$


Remark. Proposition 10 even implies that the mean value of the square of energy, $H(t)^{2}$, is uniformly bounded.

Another application is an estimate of transition probabilities under the assumption of the strong differentiability of $U_{F}(t)$. To this end we shall need the following lemma.

Lemma 11. Assume that $X, Y \in \mathscr{B}(\mathscr{H}), A$ and $B$ are bounded Hermitian operators on $\mathscr{H}$ such that

$$
A X-X B=Y \text {. }
$$

If there exist two disjoint closed intervals containing respectively $\operatorname{Spec}(A)$ and $\operatorname{Spec}(B)$ then

$$
\|X\| \leq \frac{\|Y\|}{\operatorname{dist}(\operatorname{Spec}(A), \operatorname{Spec}(B))} .
$$

Proof. For the sake of definiteness let us suppose that $\inf \operatorname{Spec}(B)>\sup \operatorname{Spec}(A)$. The solution $X$ of equation (19) is unique and given by the formula

$$
X=\frac{1}{2 \pi \mathrm{i}} \oint_{\gamma}(A-z)^{-1} Y(B-z)^{-1} d z .
$$

After a usual limit procedure we can choose for the integration path $\gamma$ in (20) the line which is parallel to the imaginary axis and intersects the real axis in the point $(\sup \operatorname{Spec}(A)+\inf \operatorname{Spec}(B)) / 2$. Integral (20) admits a simple estimate leading to the desired inequality.

Remark. Let us note that an estimate of this sort still exists when the spectra of $A$ and $B$ are interlaced provided $\operatorname{dist}(\operatorname{Spec}(A), \operatorname{Spec}(B))>0$. In the general case, as discussed in article [5], it holds true that

$$
\|X\| \leq \frac{\pi}{2} \frac{\|Y\|}{\operatorname{dist}(\operatorname{Spec}(A), \operatorname{Spec}(B))}
$$

Proposition 12. Assume that the propagator $U(t, s)$ admits a Floquet decomposition (15) which is continuously differentiable in the strong sense. Let $P(t, \cdot)$ be the projector-valued measure from the spectral decomposition of $H(t)$. Let $\Delta_{1}, \Delta_{2} \subset \mathbb{R}$ be two intervals such that $\operatorname{dist}\left(\Delta_{1}, \Delta_{2}\right)>0$. Then it holds true that

$$
\forall s, t \in \mathbb{R},\left\|P\left(t, \Delta_{1}\right) U(t, s) P\left(s, \Delta_{2}\right)\right\| \leq \frac{2\left\|S_{F}\right\|}{\operatorname{dist}\left(\Delta_{1}, \Delta_{2}\right)} .
$$

In particular, if $E_{n}(t)$ and $E_{m}(s)$ are eigenvalues of $H(t)$ and $H(s)$, respectively, $E_{n}(t) \neq E_{m}(s)$, and if $P_{n}(t)$ and $P_{m}(s)$ denote the projectors onto the corresponding eigenspaces then

$$
\left\|P_{n}(t) U(t, s) P_{m}(s)\right\| \leq \frac{2\left\|S_{F}\right\|}{\left|E_{n}(t)-E_{m}(s)\right|} .
$$


Proof. Using relation (17) one verifies the equality

$$
H(t) U(t, s)-U(t, s) H(s)=U(t, 0)\left(e^{\mathrm{i} t H_{F}} S_{F}(t) e^{-\mathrm{i} t H_{F}}-e^{\mathrm{i} s H_{F}} S_{F}(s) e^{-\mathrm{i} s H_{F}}\right) U(0, s)
$$

which is valid on $\operatorname{Dom}(H(0))$. In particular, the LHS of (21) extends to an operator bounded on $\mathscr{H}$ whose norm may be estimated from above by $2\left\|S_{F}\right\|$. Setting $A=$ $H(t) P\left(t, \Delta_{1}\right), B=H(s) P\left(s, \Delta_{2}\right)$ and $X=P\left(t, \Delta_{1}\right) U(t, s) P\left(s, \Delta_{2}\right)$, one easily finds that

$$
A X-X B=P\left(t, \Delta_{1}\right)(H(t) U(t, s)-U(t, s) H(s)) P\left(s, \Delta_{2}\right) .
$$

If the intervals $\Delta_{1}, \Delta_{2}$ are bounded then Lemma 11implies that $\|X\| \leq 2\left\|S_{F}\right\| / \operatorname{dist}\left(\Delta_{1}, \Delta_{2}\right)$. If the intervals are not bounded one can use a limit procedure.

\section{Extension: a higher order of differentiability of the Floquet decomposition}

Under assumptions on higher order differentiability in the strong sense of the operatorvalued function $U_{F}(t)$ in (15) one can extend the conclusions of Proposition 10 and Proposition 12, To this end, as an auxiliary tool we first need to state some basic facts concerning the multiple commutators.

\subsection{Multiple commutators}

Definition 13. Let $A$ be a selfadjoint operator in $\mathscr{H}, X \in \mathscr{B}(\mathscr{H})$ and $n \in \mathbb{Z}_{+}$. The sesquilinear form

$$
\alpha_{n}(\xi, \eta)=\sum_{k=0}^{n}\left(\begin{array}{l}
n \\
k
\end{array}\right)(-1)^{k}\left\langle X A^{k} \xi, A^{n-k} \eta\right\rangle_{\mathscr{H}}
$$

is well defined on $\operatorname{Dom}\left(A^{n}\right)$. If it is bounded then there exists a unique bounded operator, denoted by $\operatorname{ad}_{A}^{n} X$, such that

$$
\forall \xi, \eta \in \operatorname{Dom}\left(A^{n}\right), \alpha_{n}(\xi, \eta)=\left\langle\left(\operatorname{ad}_{A}^{n} X\right) \xi, \eta\right\rangle_{\mathscr{H}}
$$

If this is the case we shall say that (the $n$-multiple commutator) $\operatorname{ad}_{A}^{n} X$ exists in $\mathscr{B}(\mathscr{H})$.

Remark. Some elementary facts follow immediately from the definition. Suppose that $B=B^{*}$ is bounded. Then $\operatorname{ad}_{B}^{n} X \in \mathscr{B}(\mathscr{H})$ exists for all $n \in \mathbb{Z}_{+}$and it holds

$$
\operatorname{ad}_{B}^{n} X=\sum_{k=0}^{n}\left(\begin{array}{l}
n \\
k
\end{array}\right)(-1)^{k} B^{n-k} X B^{k} .
$$

Moreover, in this case $\operatorname{ad}_{A+B} X \in \mathscr{B}(\mathscr{H})$ exists if and only if $\operatorname{ad}_{A} X \in \mathscr{B}(\mathscr{H})$ exists and then $\operatorname{ad}_{A+B} X=\operatorname{ad}_{A} X+\operatorname{ad}_{B} X$. 
Definition 14. Suppose that $A=A^{*}$ in $\mathscr{H}$. For every $n \in \mathbb{Z}_{+}$we introduce the linear subspace $\mathcal{C}_{n}(A) \subset \mathscr{B}(\mathscr{H})$ formed by those bounded operators $X$ for which the commutators $\operatorname{ad}_{A}^{k} X \in \mathscr{B}(\mathscr{H})$ exist for all $k=0,1, \ldots, n$.

Remark. Clearly, $\operatorname{ad}_{A}^{0} X=X$ and $\mathcal{C}_{0}(A)=\mathscr{B}(\mathscr{H})$. From the definition it is also obvious that the vector spaces are nested, i.e.,

$$
\mathcal{C}_{0}(A) \supset \mathcal{C}_{1}(A) \supset \mathcal{C}_{2}(A) \supset \ldots
$$

Lemma 15. Suppose that $A=A^{*}$ and $X, Y \in \mathscr{B}(\mathscr{H})$. If the commutators $\operatorname{ad}_{A} X, \operatorname{ad}_{A} Y \in$ $\mathscr{B}(\mathscr{H})$ exist then there also exist $\operatorname{ad}_{A} X^{*}, \operatorname{ad}_{A}(X Y) \in \mathscr{B}(\mathscr{H})$ and it holds

(i) $X(\operatorname{Dom} A) \subset \operatorname{Dom} A$,

(ii) $\operatorname{ad}_{A} X^{*}=-\left(\operatorname{ad}_{A} X\right)^{*}$,

(iii) $\operatorname{ad}_{A}(X Y)=\left(\operatorname{ad}_{A} X\right) Y+X \operatorname{ad}_{A} Y$.

Proof. To show (ii) choose $\xi \in \operatorname{Dom} A$. By definition, for all $\eta \in \operatorname{Dom} A$ we have

$$
\langle X \xi, A \eta\rangle=\langle X A \xi, \eta\rangle+\left\langle\left(\operatorname{ad}_{A} X\right) \xi, \eta\right\rangle .
$$

Hence $X \xi$ belongs to $\operatorname{Dom} A^{*}=\operatorname{Dom} A$. Point (iii) follows from the equality

$$
\left\langle X^{*} \xi, A \eta\right\rangle-\left\langle X^{*} A \xi, \eta\right\rangle=-\overline{\langle X \eta, A \xi\rangle-\langle X A \eta, \xi\rangle}=\left\langle-\left(\operatorname{ad}_{A} X\right)^{*} \xi, \eta\right\rangle
$$

which is valid for all $\xi, \eta \in \operatorname{Dom} A$. For $\xi, \eta$ from the same domain we know, by points (ii) and (iii), that $Y \xi, X^{*} \eta \in \operatorname{Dom} A$. Thus we have the equality

$$
\langle X Y \xi, A \eta\rangle-\langle X Y A \xi, \eta\rangle=\langle X Y \xi, A \eta\rangle-\langle X A Y \xi, \eta\rangle+\left\langle Y \xi, A X^{*} \eta\right\rangle-\left\langle Y A \xi, X^{*} \eta\right\rangle
$$

with the RHS being equal to $\left\langle\left(\operatorname{ad}_{A} X\right) Y \xi, \eta\right\rangle+\left\langle\left(\operatorname{ad}_{A} Y\right) \xi, X^{*} \eta\right\rangle$. Point (iii) follows.

Remark. Lemma 15 implies that $\operatorname{ad}_{A} X \in \mathscr{B}(\mathscr{H})$ exists if and only if $\operatorname{Dom}(A)$ is invariant with respect to $X$ and the operator $A X-X A$ is bounded on this domain. If this is the case then $\operatorname{ad}_{A} X=A X-X A$ on $\operatorname{Dom}(A)$.

Lemma 16. Let $\left\{X_{n}\right\}_{n}$ be a sequence of bounded operators in $\mathscr{H}$ such that the commutators $\operatorname{ad}_{A} X_{n} \in \mathscr{B}(\mathscr{H})$ exist for all $n$. If the sequence $\left\{X_{n}\right\}_{n}$ converges weakly to a bounded operator $X$ and the sequence $\left\{\operatorname{ad}_{A} X_{n}\right\}_{n}$ converges weakly to a bounded operator $Y$ then $\operatorname{ad}_{A} X \in \mathscr{B}(\mathscr{H})$ exists and equals $Y$.

Remark. From Lemma 16 it follows that the linear operator $\operatorname{ad}_{A}$ on $\mathscr{B}(\mathscr{H})$, with $\operatorname{Dom}\left(\operatorname{ad}_{A}\right)=\mathcal{C}_{1}(A)$, is closed.

Proof. Let $\xi, \eta \in \operatorname{Dom}(A)$ be arbitrary vectors. By definition, for all $n$,

$$
\left\langle X_{n} \xi, A \eta\right\rangle-\left\langle X_{n} A \xi, \eta\right\rangle=\left\langle\left(\operatorname{ad}_{A} X_{n}\right) \xi, \eta\right\rangle .
$$

It suffices to send $n$ to infinity. 
Proposition 17. The following statements are true for all $X \in \mathscr{B}(\mathscr{H})$ and $n \in \mathbb{Z}_{+}$:

(i) If $X \in \mathcal{C}_{n}(A)$ then $X\left(\operatorname{Dom} A^{k}\right) \subset \operatorname{Dom} A^{k}$ for all $k=0,1, \ldots, n$.

(ii) $X \in \mathcal{C}_{n+1}(A)$ if and only if $\operatorname{ad}_{A} X \in \mathscr{B}(\mathscr{H})$ exists and belongs to $\mathcal{C}_{n}(A)$. Moreover, if this is the case then

$$
\operatorname{ad}_{A}^{k}\left(\operatorname{ad}_{A} X\right)=\operatorname{ad}_{A}^{k+1} X \quad \text { for } k=0,1, \ldots, n .
$$

(iii) $\mathcal{C}_{n}(A)$ is a $*$-subalgebra of $\mathscr{B}(\mathscr{H})$.

Proof. (i) We shall show that, for a given $k \in \mathbb{Z}_{+}$, the domain Dom $A^{k}$ is invariant with respect to all $X \in \mathcal{C}_{n}(A)$ as long as $n \geq k$. Recalling that the spaces $\mathcal{C}_{n}(A)$ are nested it suffices to consider the case of $n=k$. To this end, we shall proceed by induction in $k$. For $k=0$ the statement is trivial. Suppose that the statement holds true for all $\ell, 0 \leq \ell \leq k$. Choose $X \in \mathcal{C}_{k+1}(A)$. The induction hypothesis implies that for any $\xi \in \operatorname{Dom}\left(A^{k+1}\right)$ and $1 \leq \ell \leq k+1, X A^{\ell} \xi \in \operatorname{Dom}\left(A^{k+1-\ell}\right)$. By the definition of $\operatorname{ad}_{A}^{k+1} X$ we have the equality

$$
\left\langle X \xi, A^{k+1} \eta\right\rangle=\sum_{\ell=1}^{k+1}\left(\begin{array}{c}
k+1 \\
\ell
\end{array}\right)(-1)^{\ell+1}\left\langle A^{k+1-\ell} X A^{\ell} \xi, \eta\right\rangle+\left\langle\left(\operatorname{ad}_{A}^{k+1}\right) X \xi, \eta\right\rangle,
$$

valid for all $\eta \in \operatorname{Dom}\left(A^{k+1}\right)$. Hence $X \xi \in \operatorname{Dom}\left(A^{k+1}\right)$.

(ii) By the very definition, if $X \in \mathcal{C}_{n+1}(A)$ then $\operatorname{ad}_{A} X \in \mathscr{B}(\mathscr{H})$ exists. If $0 \leq m \leq$ $n$ and $\xi, \eta \in \operatorname{Dom}\left(A^{m+1}\right)$ then simple algebraic manipulations lead to the equality

$$
\sum_{k=0}^{m}\left(\begin{array}{c}
m \\
k
\end{array}\right)(-1)^{k}\left\langle\left(\operatorname{ad}_{A} X\right) A^{k} \xi, A^{m-k} \eta\right\rangle=\left\langle\left(\operatorname{ad}_{A}^{m+1} X\right) \xi, \eta\right\rangle .
$$

The both sides in (23) extend in a unique way to the domain $\xi, \eta \in \operatorname{Dom}\left(A^{m}\right)$. It follows that $\operatorname{ad}_{A}^{m}\left(\operatorname{ad}_{A} X\right) \in \mathscr{B}(\mathscr{H})$ exists and equals $\operatorname{ad}_{A}^{m+1} X$. Hence $\operatorname{ad}_{A} X \in \mathcal{C}_{n}(A)$. Conversely, suppose that $\operatorname{ad}_{A} X \in \mathcal{C}_{n}(A)$. For any $m, 0 \leq m \leq n$, and $\xi, \eta \in$ $\operatorname{Dom}\left(A^{m+1}\right)$, one finds, again with the aid of simple algebraic manipulations, that

$$
\left\langle\operatorname{ad}_{A}^{m}\left(\operatorname{ad}_{A} X\right) \xi, \eta\right\rangle=\sum_{k=0}^{m+1}\left(\begin{array}{c}
m+1 \\
k
\end{array}\right)(-1)^{k}\left\langle X A^{k} \xi, A^{m+1-k} \eta\right\rangle .
$$

Hence $\operatorname{ad}_{A}^{m+1} X \in \mathscr{B}(\mathscr{H})$ exists and thus $X \in \mathcal{C}_{n+1}(A)$.

(iii) First let us show that $X^{*} \in \mathcal{C}_{n}(A)$ provided the same is true for $X$. We shall proceed by induction in $n$. The case $n=0$ is obvious. Suppose that the claim is true for $n$. If $X \in \mathcal{C}_{n+1}(A)$ then, by the already proved point (iii) of the current proposition, $\operatorname{ad}_{A} X \in \mathcal{C}_{n}(A)$. By the induction hypothesis and Lemma 15 ad (iii) we have $\operatorname{ad}_{A} X^{*}=-\left(\operatorname{ad}_{A} X\right)^{*} \in \mathcal{C}_{n}(A)$. Referring once more to point (iii) of the current proposition we conclude that indeed $X^{*} \in \mathcal{C}_{n+1}(A)$.

Finally let us show that $X Y \in \mathcal{C}_{n}(A)$ provided $X, Y \in \mathcal{C}_{n}(A)$. We shall proceed by induction in $n$. The case $n=0$ is again obvious. Suppose that the claim is true for $n$. 
If $X, Y \in \mathcal{C}_{n+1}(A)$ then, by point (ii) of the current proposition, $\operatorname{ad}_{A} X, \operatorname{ad}_{A} Y \in \mathcal{C}_{n}(A)$. By the induction hypothesis and Lemma [15] ad (iii) we have

$$
\operatorname{ad}_{A}(X Y)=\left(\operatorname{ad}_{A} X\right) Y+X \operatorname{ad}_{A} Y \in \mathcal{C}_{n}(A) .
$$

Referring again to point (iii) of the current proposition we conclude that $X Y \in$ $\mathcal{C}_{n+1}(A)$.

Remark. As an immediate consequence of Proposition [17 ad (ii) it holds $\operatorname{Dom}\left(A^{k}\right)=$ $\operatorname{Dom}\left((A+B)^{k}\right)$ for $k=0,1, \ldots, p$, provided $B \in \mathcal{C}_{p-1}(A)$ for some $p \in \mathbb{N}$.

Definition 18. Let $A$ be a self-adjoint operator on $\mathscr{H}$ and $X(t) \in \mathscr{B}(\mathscr{H})$ be an operator-valued function, with the variable $t$ running over $\mathbb{R}$, and let $n \in \mathbb{Z}_{+}$. We shall say that $X(t)$ is in the algebra $\mathcal{C}_{n}(A)$ uniformly if $X(t) \in \mathcal{C}_{n}(A)$ for all $t \in \mathbb{R}$ and

$$
\sup _{t \in \mathbb{R}} \sum_{k=0}^{n}\left\|\operatorname{ad}_{A}^{k} X(t)\right\|<\infty .
$$

Remarks. Of course, the operator-valued function $X(t)$ may be constant.

From Proposition 17 ad (iil) one immediately deduces that an operator-valued function $X(t) \in \mathscr{B}(\mathscr{H})$ is in $\mathcal{C}_{n+1}(A)$ uniformly if and only if $X(t)$ is uniformly bounded and $\operatorname{ad}_{A} X(t)$ is in $\mathcal{C}_{n}(A)$ uniformly. Moreover, a straightforward induction procedure based on this observation jointly with Lemma 15 ad (iii) and ad (iii) implies that if $X(t)$ and $Y(t)$ are in $\mathcal{C}_{n}(A)$ uniformly then also $X(t)^{*}$ and $X(t) Y(t)$ are in $\mathcal{C}_{n}(A)$ uniformly.

Lemma 19. Let $A$ be a self-adjoint operator and $B \in \mathcal{C}_{p-1}(A)$ be a Hermitian operator for some $p \in \mathbb{N}$. Then an operator-valued function $X(t) \in \mathscr{B}(\mathscr{H})$, with $t \in \mathbb{R}$, is in $\mathcal{C}_{p}(A)$ uniformly if and only if $X(t)$ is in $\mathcal{C}_{p}(A+B)$ uniformly.

Proof. Clearly it suffices to prove only one implication since the other one follows after replacing $A$ by $A+B$ and $B$ by $-B$ (while making use of the simple fact that $\left.\operatorname{ad}_{A+B}^{m} B=\operatorname{ad}_{A}^{m} B\right)$. We shall proceed by induction in $p$.

As far as the case $p=1$ is concerned we assume that $B \in \mathcal{C}_{0}(A)$ and $X(t)$ is in $\mathcal{C}_{1}(A)$ uniformly. This in particular means that $X(t)$ is a uniformly bounded operatorvalued function and hence the same is true for $\operatorname{ad}_{B} X(t)=B X(t)-X(t) B$. Now it suffices to take into account the equality $\operatorname{ad}_{A+B} X(t)=\operatorname{ad}_{A} X(t)+\operatorname{ad}_{B} X(t)$.

Let us now assume that the lemma has been proved for some $p \in \mathbb{N}$, and that $B \in \mathcal{C}_{p}(A)$ and $X(t)$ is in $\mathcal{C}_{p+1}(A)$ uniformly. Now we can repeatedly apply the remarks following Definition [18. Firstly, $\operatorname{ad}_{B} X(t)=B X(t)-X(t) B$ is in $\mathcal{C}_{p}(A)$ uniformly. Secondly, $X(t)$ is uniformly bounded and $\operatorname{ad}_{A} X(t)$ is in $\mathcal{C}_{p}(A)$ uniformly. Consequently, $\operatorname{ad}_{A+B} X(t)$ is in $\mathcal{C}_{p}(A)$ uniformly as well. By the induction hypothesis, $\operatorname{ad}_{A+B} X(t)$ is in $\mathcal{C}_{p}(A+B)$ uniformly. This in turn implies that $X(t)$ is in $\mathcal{C}_{p+1}(A+B)$ uniformly.

In the particular case when the operator-valued function $X(t)$ is constant Lemma 19 reduces to the following statement. 
Lemma 20. Let $A$ be a self-adjoint operator on $\mathscr{H}$ and $B \in \mathcal{C}_{p-1}(A)$ for some $p \in \mathbb{N}$, and suppose that $B=B^{*}$. Then $\mathcal{C}_{k}(A)=\mathcal{C}_{k}(A+B)$ for $k=0,1, \ldots, p$.

We shall also need the following algebraic lemma.

Lemma 21. Suppose that $A=A^{*}$ and $B \in \mathcal{C}_{p}(A)$ for some $p \in \mathbb{Z}_{+}$. Then the following claims are true:

(i) On $\operatorname{Dom}\left(A^{p}\right)$ it holds

$$
A^{p} B=\sum_{k=0}^{p}\left(\begin{array}{l}
p \\
k
\end{array}\right)\left(\operatorname{ad}_{A}^{p-k} B\right) A^{k}
$$

(ii) There exist polynomials $F_{p, k}\left(\mathfrak{x}_{0}, \mathfrak{x}_{1}, \ldots, \mathfrak{x}_{p-k-1}\right), k=0,1, \ldots, p-1$, in non-commutative variables $\mathfrak{x}_{j}$, with non-negative integer coefficients and such that it holds

$$
(A+B)^{p}=A^{p}+\sum_{k=0}^{p-1} F_{p, k}\left(B, \operatorname{ad}_{A} B, \ldots, \operatorname{ad}_{A}^{p-k-1} B\right) A^{k}
$$

on $\operatorname{Dom}\left(A^{p}\right)$.

Proof. (i) By Proposition 17 ad (ii) the both sides of (24) are well defined on $\operatorname{Dom}\left(A^{p}\right)$. To verify (24) one can proceed by induction in $p$ which amounts to simple algebraic manipulations. We omit the details.

(ii) Again, the both sides of (25) are well defined on $\operatorname{Dom}\left(A^{p}\right)$. One can proceed by induction in $p$. Set, by convention, $F_{p, p}=1$. To carry out the induction step let us write

$$
(A+B)^{p+1}=\sum_{k=0}^{p} F_{p, k}\left(B, \operatorname{ad}_{A} B, \ldots, \operatorname{ad}_{A}^{p-k-1} B\right) A^{k}(A+B)
$$

and apply claim (ii) of the current lemma to manage the term $A^{k} B$ on the RHS. By comparison one arrives at the recursion rule

$$
F_{p+1, k}\left(\mathfrak{x}_{0}, \mathfrak{x}_{1}, \ldots, \mathfrak{x}_{p-k}\right)=F_{p, k-1}\left(\mathfrak{x}_{0}, \mathfrak{x}_{1}, \ldots, \mathfrak{x}_{p-k}\right)+\sum_{\ell=k}^{p}\left(\begin{array}{l}
\ell \\
k
\end{array}\right) F_{p, \ell}\left(\mathfrak{x}_{0}, \mathfrak{x}_{1}, \ldots, \mathfrak{x}_{p-\ell-1}\right) \mathfrak{x}_{\ell-k}
$$

from which claim (iii) easily follows.

\subsection{Differentiable Floquet decompositions}

In this section we shall assume that

$$
V(t):=H(t)-H(0)
$$

is a uniformly bounded operator-valued function. Of course, it is Hermitian and Tperiodic. We also assume that we are given a Floquet decomposition (15) of the corresponding propagator $U(t, s)$. For $p \in \mathbb{Z}_{+}$let us set

$$
\mathcal{A}_{p}^{0}=\mathcal{C}_{p}\left(H_{0}\right), \mathcal{A}_{p}=\mathcal{C}_{p}\left(H_{F}\right) .
$$


Here and everywhere in this section we write shortly $H_{0}=H(0)$ and $S_{0}=S_{F}(0)$. Thus we have $H_{0}=H_{F}+S_{0}$ (see (16) and (17)).

If the Floquet decomposition is continuously differentiable in the strong sense and $S_{0} \in \mathcal{A}_{p-1}$ for some $p \in \mathbb{N}$ then Lemma 20 tells us that $\mathcal{A}_{k}=\mathcal{A}_{k}^{0}$ for $k=0,1, \ldots, p$.

Lemma 22. Let us assume that $p \in \mathbb{N}$ and $V(t) \in C^{p-1}(\mathbb{R})$ in the strong sense, and that the propagator $U(t, s)$ admits a Floquet decomposition (15) which is $p$ times continuously differentiable in the strong sense. If

$$
V^{(k)}(t) \text { is in } \mathcal{A}_{p-1-k} \text { uniformly for } k=0,1, \ldots, p-1 \text {, }
$$

then

$$
U_{F}^{(k)}(t) \text { is in } \mathcal{A}_{p-k} \text { uniformly for } k=0,1, \ldots, p \text {. }
$$

Moreover, $\mathcal{A}_{k}=\mathcal{A}_{k}^{0}$ for $k=0,1, \ldots, p$, and $S_{F}(t)=\mathrm{i} U_{F}(t)^{-1} \partial_{t} U_{F}(t)$ is in $\mathcal{A}_{p-1}$ uniformly.

Proof. For the proof we shall need the relation

$$
\operatorname{ad}_{H_{F}} U_{F}(t)=U_{F}(t) S_{F}(t)-\left(S_{0}+V(t)\right) U_{F}(t) .
$$

Here $U_{F}(t)$ preserves the domain $\operatorname{Dom}\left(H_{F}\right)=\operatorname{Dom}\left(H_{0}\right)$. Equality (28) follows from (17) and the substitution

$$
H(t)=H_{0}+V(t)=S_{0}+H_{F}+V(t) .
$$

From the differentiability of $U_{F}(t)$ it follows that $S_{F}(t)$ belongs to $C^{p-1}(\mathbb{R})$ in the strong sense. Thus all derivatives of $S_{F}(t)$ up to the order $p-1$ are uniformly bounded (due to the periodicity). With the aid of Lemma 16 we derive from (28) that

$$
\operatorname{ad}_{H_{F}} U_{F}^{(k)}(t)=\frac{\mathrm{d}^{k}}{\mathrm{~d} t^{k}}\left(U_{F}(t) S_{F}(t)-\left(S_{0}+V(t)\right) U_{F}(t)\right) \in \mathscr{B}(\mathscr{H}) \text { for } k=0,1, \ldots, p-1,
$$

(with all derivatives taken in the strong sense). Moreover, $\operatorname{ad}_{H_{F}} U_{F}^{(k)}(t)$ is uniformly bounded for $0 \leq k \leq p-1$. Note also that (26) can be rewritten in the form

$$
V^{(k)}(t) \text { is in } \mathcal{A}_{p-1-\ell} \text { uniformly if } 0 \leq k \leq \ell \leq p-1
$$

since the algebras $\mathcal{A}_{r}$ are nested, $\mathcal{A}_{0} \supset \mathcal{A}_{1} \supset \mathcal{A}_{2} \supset \ldots$, (see (22)).

We shall verify that, for $\ell=0,1, \ldots, p$,

$$
U_{F}^{(k)}(t) \text { is in } \mathcal{A}_{p-\ell} \text { uniformly if } 0 \leq k \leq \ell \leq p .
$$

Since

$$
S_{F}^{(k)}(t)=\frac{\mathrm{d}^{k}}{\mathrm{~d} t^{k}}\left(\mathrm{i} U_{F}(t)^{*} U_{F}^{\prime}(t)\right)
$$

and $\mathcal{A}_{n}$ is a $*$-algebra relation (31) implies that, for $\ell=1, \ldots, p$,

$$
S_{F}^{(k)}(t) \text { is in } \mathcal{A}_{p-\ell} \text { uniformly if } 0 \leq k \leq \ell-1 \leq p-1 .
$$


To show (31) we shall proceed by a finite descending induction in $\ell$. According to the assumptions of the lemma, $U_{F}^{(k)}(t)$ is uniformly bounded for $0 \leq k \leq p$ and the case $\ell=p$ follows. Suppose now that (31) is valid for some $\ell, 1 \leq \ell \leq p$. Then for the same $\ell$, (32) is valid as well. Moreover, replacing $\ell$ by $\ell-1$ in (30) one knows that $V^{(k)}(t)$ is in $\mathcal{A}_{p-\ell}$ uniformly for $0 \leq k \leq \ell-1$. Thus if $0 \leq k \leq \ell-1$ then from the fact that $\mathcal{A}_{p-\ell}$ is an algebra and from the induction hypothesis one deduces that the RHS of (29) is in $\mathcal{A}_{p-\ell}$ uniformly. This implies, in virtue of Proposition $17 \mathrm{ad}$ (iii), that $U_{F}^{(k)}(t)$ is in $\mathcal{A}_{p-\ell+1}$ uniformly. This completes the induction step and relation (31) is verified.

Setting $k=\ell$ in (31) one obtains (27). Setting $k=0$ and $\ell=1$ in (32) one finds that $S_{F}(t)$ is $\mathcal{A}_{p-1}$ uniformly. In particular, $S_{0} \equiv S_{F}(0)$ belongs to $\mathcal{A}_{p-1}=\mathcal{C}_{p-1}\left(H_{F}\right)$. Since $H_{0}=H_{F}+S_{0}$ from Lemma 20 we know that

$$
\mathcal{A}_{k}=\mathcal{C}_{k}\left(H_{F}\right)=\mathcal{C}_{k}\left(H_{0}\right)=\mathcal{A}_{k}^{0}
$$

for $k=0,1, \ldots, p$.

Corollary 23. Lemma 22 remains still true if $\mathcal{A}_{p-1-k}$ is replaced by $\mathcal{A}_{p-1-k}^{0}$ in the condition (26).

Proof. We shall proceed by induction in $p$. For $p=1$ we have $\mathcal{A}_{0}^{0}=\mathcal{A}_{0}=\mathscr{B}(\mathscr{H})$ and thus replacing $\mathcal{A}_{p-1-k}$ by $\mathcal{A}_{p-1-k}^{0}$ in (26) does not mean any change. Let us now suppose that the claim is true for some $p \in \mathbb{N}$. And we assume that $V^{(k)}(t)$ is in $\mathcal{A}_{p-k}^{0}$ uniformly for $k=0,1, \ldots, p$. Of course, the other assumptions of Lemma 22, except of the condition (26), are satisfied as well, namely $V(t) \in C^{p}(\mathbb{R})$ in the strong sense, and the propagator $U(t, s)$ admits a Floquet decomposition (15) which is $p+1$ times continuously differentiable in the strong sense. Since $\mathcal{A}_{p-k}^{0} \subset \mathcal{A}_{p-1-k}^{0}, V^{(k)}(t)$ is in $\mathcal{A}_{p-1-k}^{0}$ uniformly for $k=0,1, \ldots, p-1$. By the induction hypothesis, Lemma 22 is applicable for the value $p$ and therefore, in particular, $\mathcal{A}_{k}=\mathcal{A}_{k}^{0}$ for $k=0,1, \ldots, p$. Hence $V^{(k)}(t)$ is in $\mathcal{A}_{p-k}$ uniformly for $k=0,1, \ldots, p$, which is nothing but condition (26) with $p$ being replaced by $p+1$. It follows that the conclusions of Lemma 22 hold true for the value $p+1$ as well.

Proposition 24. Let us assume that $p \in \mathbb{N}$ and $V(t) \in C^{p-1}(\mathbb{R})$ in the strong sense, and that the propagator $U(t, s)$ admits a Floquet decomposition (15) which is p times continuously differentiable in the strong sense. If

$$
V^{(k)}(t) \text { is in } \mathcal{A}_{p-1-k}^{0} \text { uniformly for } k=0,1, \ldots, p-1,
$$

then $U(t, 0), t \in \mathbb{R}$, preserves the domain $\operatorname{Dom}\left(H_{0}^{p}\right)$ and

$$
\forall \psi \in \operatorname{Dom}\left(H_{0}^{p}\right), \sup _{t \in \mathbb{R}}\left\|H(t)^{p} U(t, 0) \psi\right\|<\infty .
$$

Proof. From Corollary 23 we know that $U_{F}(t)$ is in $\mathcal{A}_{p}$ uniformly and $S_{F}(t)$ is in $\mathcal{A}_{p-1}$ uniformly. Since $S_{0} \in \mathcal{A}_{p-1}$ and $H_{0}=H_{F}+S_{0}$, Lemma 20 tells us that $\mathcal{A}_{k}=\mathcal{A}_{k}^{0}$ for $0 \leq k \leq p$. From the relations $V(t) \in \mathcal{A}_{p-1}^{0}$ and $S_{0} \in \mathcal{A}_{p-1}$ it also follows that

$$
\operatorname{Dom}\left(H_{0}^{k}\right)=\operatorname{Dom}\left(H(t)^{k}\right)=\operatorname{Dom}\left(H_{F}^{k}\right) \text { for } k=0,1, \ldots, p,
$$


see Proposition 17 ad (ii). Furthermore, from the Floquet decomposition (15) and the above observation on $U_{F}(t)$ one deduces that $U(t, 0)$ is in $\mathcal{A}_{p}=\mathcal{A}_{p}^{0}$ uniformly and therefore $U(t, 0)\left(\operatorname{Dom}\left(H_{0}^{p}\right)\right) \subset \operatorname{Dom}\left(H_{0}^{p}\right)$.

Suppose that $\psi \in \operatorname{Dom}\left(H_{0}^{p}\right)$. From (15) and (17) one finds that

$$
H(t)^{p} U(t, 0) \psi=U_{F}(t)\left(H_{F}+S_{F}(t)\right)^{p} e^{-\mathrm{i} t H_{F}} \psi .
$$

With the aid of equality (25) of Lemma 21 ad (iii) one derives the estimate

$$
\left\|H(t)^{p} U(t, 0) \psi\right\| \leq \sum_{k=0}^{p} F_{p, k}\left(\mathfrak{S}_{0}, \mathfrak{S}_{1}, \ldots, \mathfrak{S}_{p-k-1}\right)\left\|H_{F}^{k} \psi\right\|
$$

where

$$
\mathfrak{S}_{k}:=\sup _{t \in \mathbb{R}}\left\|\operatorname{ad}_{H_{F}}^{k} S_{F}(t)\right\|, k=0,1, \ldots, p-1 .
$$

The proposition follows.

Lemma 25. Under the same assumptions as in Proposition 24, the operators

$$
X_{n}(t, s)=\sum_{k=0}^{n}\left(\begin{array}{l}
n \\
k
\end{array}\right)(-1)^{k} H(t)^{n-k} U(t, s) H(s)^{k}, \quad n=0,1, \ldots, p,
$$

are well defined on $\operatorname{Dom}\left(H_{0}^{n}\right)$. Moreover, $X_{n}(t, s)$ extends in a unique way to a bounded operator on $\mathscr{H}$ which is in $\mathcal{A}_{p-n}$ uniformly with respect to the variables $(t, s) \in \mathbb{R}^{2}$.

Proof. In the same way as in the proof of Proposition 24, we deduce from the assumptions that equalities (34) hold true as well as that $U_{F}(t)$ is in $\mathcal{A}_{p}=\mathcal{A}_{p}^{0}$ uniformly and $S_{F}(t)$ is in $\mathcal{A}_{p-1}$ uniformly. Moreover, Proposition 17 ad (ii) tells us that $U_{F}(t)$ preserves $\operatorname{Dom}\left(H_{F}^{k}\right)$ for $k=0,1, \ldots, p$.

From (15) and (17) it follows that

$$
X_{n}(t, s)=U_{F}(t) Z_{n}(t, s) U_{F}(s)^{-1}
$$

where

$$
Z_{n}(t, s)=\sum_{k=0}^{n}\left(\begin{array}{l}
n \\
k
\end{array}\right)(-1)^{k}\left(H_{F}+S_{F}(t)\right)^{n-k} e^{-\mathrm{i}(t-s) H_{F}}\left(H_{F}+S_{F}(s)\right)^{k} .
$$

It suffices to show that $Z_{n}(t, s)$ is well defined on $\operatorname{Dom}\left(H_{F}^{n}\right)$ and extends to a bounded operator on $\mathscr{H}$ which is in $\mathcal{A}_{p-n}$ uniformly. To verify it we proceed by induction in $n$.

For $n=0, Z_{0}(t, s)=e^{-\mathrm{i}(t-s) H_{F}}$ fulfills $\operatorname{ad}_{H_{F}}^{k} Z_{0}(t, s)=0$ for all $k \geq 1$ and so it is in $\mathcal{A}_{p}$ uniformly. To carry out the induction step observe that

$$
\begin{aligned}
Z_{n+1}(t, s) & =\left(H_{F}+S_{F}(t)\right) Z_{n}(t, s)-Z_{n}(t, s)\left(H_{F}+S_{F}(s)\right) \\
& =\operatorname{ad}_{H_{F}} Z_{n}(t, s)+S_{F}(t) Z_{n}(t, s)-Z_{n}(t, s) S_{F}(s) .
\end{aligned}
$$

The induction hypothesis and Proposition 17 ad (iii) (see also Remarks following Definition (18) imply that $\operatorname{ad}_{H_{F}} Z_{n}(t, s)$ is in $\mathcal{A}_{p-n-1}$ uniformly. Recalling Proposition 17 ad (iii) it also holds true that $S_{F}(t) Z_{n}(t, s)$ and $Z_{n}(t, s) S_{F}(s)$ are in $\mathcal{A}_{p-n-1}$ uniformly. This verifies the induction step and concludes the proof of the lemma. 
Proposition 26. Under the same assumptions as in Proposition 24 (including condition (33)), let $P(t, \cdot)$ be the projection-valued measure from the spectral decomposition of $H(t)$. Then there exists a constant $C_{p} \geq 0$ such that for any couple of intervals $\Delta_{1}, \Delta_{2} \subset \mathbb{R}$ whose distance $\operatorname{dist}\left(\Delta_{1}, \Delta_{2}\right)$ is positive it holds true

$$
\forall s, t \in \mathbb{R},\left\|P\left(t, \Delta_{1}\right) U(t, s) P\left(s, \Delta_{2}\right)\right\| \leq \frac{C_{p}}{\operatorname{dist}\left(\Delta_{1}, \Delta_{2}\right)^{p}} .
$$

Proof. It suffices to verify the assertion for bounded intervals. The general case then follows by a limit procedure. Set $Y_{n}(t, s)=P\left(t, \Delta_{1}\right) X_{n}(t, s) P\left(s, \Delta_{2}\right)$ where $X_{n}(t, s)$ is defined in (35), and $Q_{1}(t)=H(t) P\left(t, \Delta_{1}\right), Q_{2}(s)=H(s) P\left(s, \Delta_{2}\right)$. In particular, $Y_{0}(t, s)=P\left(t, \Delta_{1}\right) U(t, s) P\left(s, \Delta_{2}\right)$. From Lemma 25 we know that the operator-valued functions $X_{n}(t, s)$ are uniformly bounded. If $0 \leq n<p$ then it holds

$$
Q_{1}(t) Y_{n}(t, s)-Y_{n}(t, s) Q_{2}(s)=Y_{n+1}(t, s) .
$$

By Lemma 11 we have the estimate

$$
\left\|Y_{n}(t, s)\right\| \leq \frac{\left\|Y_{n+1}(t, s)\right\|}{\operatorname{dist}\left(\operatorname{Spec}\left(Q_{1}(t)\right), \operatorname{Spec}\left(Q_{2}(s)\right)\right)} \leq \frac{\left\|Y_{n+1}(t, s)\right\|}{\operatorname{dist}\left(\Delta_{1}, \Delta_{2}\right)} .
$$

Applying this estimate consecutively for $n=0,1, \ldots, p-1$, we find that (36) holds true with $C_{p}=\sup _{(t, s) \in \mathbb{R}^{2}}\left\|X_{p}(t, s)\right\|$.

\section{A solvable example: the time-dependent har- monic oscillator}

Let us consider the time-dependent harmonic oscillator

$$
H(t)=H_{\omega}+f(t) x, H_{\omega}=-\frac{1}{2} \partial_{x}^{2}+\frac{\omega^{2} x^{2}}{2},
$$

in $\mathscr{H}=L^{2}(\mathbb{R}, \mathrm{d} x)$ where the function $f(t)$ is supposed to be continuous and $T$ periodic. The Hamiltonians quadratic in $x$ and $p$ turn out to be quite attractive in various situations since they allow for explicit computations. For example, a classical result is a formula for the Green function computed in the framework of the Feynman path integral [14], see also [23] and comments on the literature therein. For purposes of the present paper we need some of the results derived in [13] and concerned with the dynamical properties of $H(t)$, see also an additional analysis in [6, Chp. 5]. Let us also mention that in [15] it has been shown that the Floquet operator associated to a time-dependent quadratic Hamiltonian can only have either a pure point spectrum or a purely absolutely transient continuous spectrum.

As pointed out in [13], it holds

$$
\begin{aligned}
& U(t, 0)^{-1} x U(t, 0)=x \cos (\omega t)+\frac{p}{\omega} \sin (\omega t)-\frac{1}{\omega} \varphi_{2}(t, 0), \\
& U(t, 0)^{-1} p U(t, 0)=-\omega x \sin (\omega t)+p \cos (\omega t)+\varphi_{1}(t, 0),
\end{aligned}
$$


where the functions $\varphi_{1}(t, s)$ and $\varphi_{2}(t, s)$ are given in (A.9). Assume for a moment that $\varphi_{1}(t, 0)$ and $\varphi_{2}(t, 0)$ are uniformly bounded. Under this assumption it is obvious that if an initial condition $\psi$ belongs to the Schwartz space $\mathscr{S}$ then the quantity $\langle U(t, 0) \psi, P(p, x) U(t, 0) \psi\rangle$ is uniformly bounded in time for any polynomial $P(p, x)$ in the non-commuting variables $p=-\mathrm{i} \partial_{x}$ and $x$. In particular, for such an initial condition, the mean value of energy is bounded uniformly. As stated in [13, Proposition 4.1], it follows that all trajectories $\{U(t, 0) \psi ; t \in \mathbb{R}\}$, for any initial condition $\psi \in \mathscr{H}$, are precompact subsets in $\mathscr{H}$. This in turn implies that the spectrum of the monodromy operator $U(T, 0)$ is pure point (see Theorem 2.3 in [13]). The fact that the mean value of energy is bounded for all initial conditions from a total set has also the following consequence (see Lemma 3.3 in [13]):

$$
\forall \psi \in \mathscr{H}, \lim _{R \rightarrow \infty} \sup _{t \in \mathbb{R}}\left\|F\left(H_{\omega}>R\right) U(t, 0) \psi\right\|=0
$$

where the symbol $F$ stands for the projection-valued measure from the spectral decomposition of the operator indicated in the argument and taken for a subset of the real line which is indicated in the argument as well.

Let us note that paper [13] has finally focused on the particular case $f(t)=$ $\sin (2 \pi t / T)$. In that case a simple computation shows that the functions $\varphi_{1}(t, 0)$ and $\varphi_{2}(t, 0)$ are bounded if and only if $2 \pi / T \neq \omega$.

Let us now examine how Proposition 10 can be applied to this example. We consider the non-resonant case

$$
T \notin \frac{2 \pi}{\omega} \mathbb{N} .
$$

Let us write

$$
\left.T=\frac{2 \pi}{\omega} N+\Delta, \text { with } N \in \mathbb{Z}_{+}, \Delta \in\right] 0, \frac{2 \pi}{\omega}[.
$$

As a first step one has to make a choice of a self-adjoint operator $H_{F}$ so that $U(T, 0)=$ $\exp \left(-\mathrm{i} T H_{F}\right)$. According to Proposition A.3, the monodromy operator corresponding to $H(t)$ can be expressed in the form

$$
U(T, 0)=(-1)^{N} \exp \left(-\mathrm{i} \Delta H_{\omega}+\mathrm{i} \frac{\mu(T, 0)}{\omega} p+\mathrm{i} \nu(T, 0) x+\mathrm{i} \sigma(T, 0)\right)
$$

where the functions $\mu(t, s)$ and $\nu(t, s)$ are given in (A.12) and $\sigma(t, s)$ is given in (A.13).

We shall seek $H_{F}$ in the form

$$
H_{F}=H_{\omega}-\frac{\alpha}{\omega T} p-\frac{\beta}{T} x+\frac{\gamma}{T}
$$

for some $\alpha, \beta, \gamma \in \mathbb{R}$. Then it holds

$$
\begin{aligned}
\exp \left(-\mathrm{i} T H_{F}\right)= & e^{-\mathrm{i} \gamma} \exp \left(-\mathrm{i} T H_{\omega}+\mathrm{i} \frac{\alpha}{\omega} p+\mathrm{i} \beta x\right) \\
= & \exp \left(-\mathrm{i} \gamma+\mathrm{i} \frac{\alpha^{2}+\beta^{2}}{2 \omega^{2} T}\right) \exp \left(\mathrm{i} \frac{\alpha}{\omega T} x\right) \exp \left(-\mathrm{i} \frac{\beta}{\omega^{2} T} p\right) \exp \left(-\mathrm{i} T H_{\omega}\right) \\
& \times \exp \left(\mathrm{i} \frac{\beta}{\omega^{2} T} p\right) \exp \left(-\mathrm{i} \frac{\alpha}{\omega T} x\right)
\end{aligned}
$$


Here we have used that

$$
e^{\mathrm{i} s x} H_{\omega} e^{-\mathrm{i} s x}=H_{\omega}-s p+\frac{s^{2}}{2}, e^{\mathrm{i} s p} H_{\omega} e^{-\mathrm{i} s p}=H_{\omega}+s \omega^{2} x+\frac{s^{2} \omega^{2}}{2} .
$$

By the well known spectral properties of $H_{\omega}, \exp \left(-\mathrm{i} T H_{\omega}\right)$ equals $(-1)^{N} \exp \left(-\mathrm{i} \Delta H_{\omega}\right)$, and so one finally arrives at the expression

$$
(-1)^{N} \exp \left(-\mathrm{i} \gamma+\mathrm{i} \frac{\alpha^{2}+\beta^{2}}{2 \omega^{2} T}\left(1-\frac{\Delta}{T}\right)\right) \exp \left(-\mathrm{i} \Delta H_{\omega}+\mathrm{i} \frac{\alpha \Delta}{\omega T} p+\mathrm{i} \frac{\beta \Delta}{T} x\right) .
$$

Equating this expression to the RHS of (37) one has to set

$$
\alpha=\frac{T}{\Delta} \mu(t, 0), \beta=\frac{T}{\Delta} \nu(t, 0),-\gamma+\frac{\alpha^{2}+\beta^{2}}{2 \omega^{2} T}\left(1-\frac{\Delta}{T}\right)=\sigma(T, 0) .
$$

Thus our choice of $H_{F}$ reads

$$
H_{F}=H_{\omega}-\frac{\mu(T, 0)}{\omega \Delta} p-\frac{\nu(T, 0)}{\Delta} x-\frac{\sigma(T, 0)}{T}+\pi N \frac{\mu(T, 0)^{2}+\nu(T, 0)^{2}}{\omega^{3} \Delta^{2} T} .
$$

As a next step one has to compute the $T$-periodic family of unitary operators $U_{F}(t)=U(t, 0) \exp \left(\mathrm{i} t H_{F}\right)$. With the aid of Lemma A.1 one can express

$$
\begin{aligned}
\exp \left(-\mathrm{i} t H_{F}\right)= & \exp \left(-\mathrm{i} \phi(t)+\mathrm{i} \frac{\sigma(T, 0) t}{T}-\mathrm{i} \pi N \frac{\left(\mu(T, 0)^{2}+\nu(T, 0)^{2}\right) t}{\omega^{3} \Delta^{2} T}\right) \\
& \times \exp \left(\mathrm{i} \frac{\xi(t)}{\omega} p\right) \exp (\mathrm{i} \eta(t) x) \exp \left(-\mathrm{i} t H_{\omega}\right)
\end{aligned}
$$

where

$$
\begin{aligned}
& \xi(t)=\frac{2}{\omega \Delta} \sin \left(\frac{\omega t}{2}\right)\left(\cos \left(\frac{\omega t}{2}\right) \mu(T, 0)-\sin \left(\frac{\omega t}{2}\right) \nu(T, 0)\right), \\
& \eta(t)=\frac{2}{\omega \Delta} \sin \left(\frac{\omega t}{2}\right)\left(\sin \left(\frac{\omega t}{2}\right) \mu(T, 0)+\cos \left(\frac{\omega t}{2}\right) \nu(T, 0)\right),
\end{aligned}
$$

and

$$
\begin{aligned}
\phi(t)= & -\frac{1}{4 \omega^{3} \Delta^{2}}\left((2 \omega t-4 \sin (\omega t)+\sin (2 \omega t)) \mu(T, 0)^{2}\right. \\
& \left.+(2-4 \cos (\omega t)+2 \cos (2 \omega t)) \mu(T, 0) \nu(T, 0)+(2 \omega t-\sin (2 \omega t)) \nu(T, 0)^{2}\right) .
\end{aligned}
$$

Using relations (A.12) for $\mu(T, 0)$ and $\nu(T, 0)$ this can be rewritten as

$$
\begin{aligned}
\xi(t) & =\frac{\sin \left(\frac{\omega t}{2}\right)}{\sin \left(\frac{\omega T}{2}\right)} \int_{0}^{T} \sin \left(\omega\left(\frac{t+T}{2}-u\right)\right) f(u) \mathrm{d} u \\
\eta(t) & =-\frac{\sin \left(\frac{\omega t}{2}\right)}{\sin \left(\frac{\omega T}{2}\right)} \int_{0}^{T} \cos \left(\omega\left(\frac{t+T}{2}-u\right)\right) f(u) \mathrm{d} u
\end{aligned}
$$


and it also holds true that (compare to (A.7))

$$
\phi(t)=\frac{1}{2 \omega} \xi(t) \eta(t)-\frac{\omega t-\sin (\omega t)}{8 \omega \sin \left(\frac{\omega t}{2}\right)^{2}}\left(\xi(t)^{2}+\eta(t)^{2}\right) .
$$

Expressing the propagator $U(t, 0)$ according to formula (A.8) due to Enss and Veselic̀ one finally arrives at the sought equality

$$
U_{F}(t)=e^{\mathrm{i} \Phi(t)} e^{\mathrm{i} F_{2}(t) x} e^{\mathrm{i}\left(F_{1}(t) / \omega\right) p}
$$

where

$$
F_{1}(t)=\varphi_{2}(t, 0)-\xi(t), F_{2}(t)=-\varphi_{1}(t, 0)-\eta(t)
$$

and

$$
\Phi(t)=-\psi(t, 0)+\phi(t)-\frac{\sigma(T, 0) t}{T}+\pi N \frac{\left(\mu(T, 0)^{2}+\nu(T, 0)^{2}\right) t}{\omega^{3} \Delta^{2} T}-\frac{\varphi_{2}(t, 0) \eta(t)}{\omega}
$$

$(\psi(t, s)$ is given in $(\underline{A .10}))$. After some elementary manipulations this can be rewritten as

$$
\begin{aligned}
& F_{1}(t)=\frac{1}{2 \sin \left(\frac{\omega T}{2}\right)} \int_{0}^{T} \cos \left(\omega\left(u-\frac{T}{2}\right)\right)(f(t-u)-f(u)) \mathrm{d} u \\
& F_{2}(t)=\frac{1}{2 \sin \left(\frac{\omega T}{2}\right)} \int_{0}^{T} \sin \left(\omega\left(u-\frac{T}{2}\right)\right)(f(t-u)+f(u)) \mathrm{d} u
\end{aligned}
$$

and

$$
\begin{aligned}
\Phi(t)= & -\frac{1}{2} \int_{0}^{t}\left(\varphi_{1}(v, 0)^{2}-\varphi_{2}(v, 0)^{2}\right) \mathrm{d} v+\frac{t}{2 T} \int_{0}^{T}\left(\varphi_{1}(v, 0)^{2}-\varphi_{2}(v, 0)^{2}\right) \mathrm{d} v \\
& +\frac{1}{2 \omega} \xi(t) \eta(t)-\frac{t}{2 \omega T} \varphi_{1}(T, 0) \varphi_{2}(T, 0)-\frac{\varphi_{2}(t, 0) \eta(t)}{\omega} \\
& -\frac{\omega t-\sin (\omega t)}{8 \omega \sin \left(\frac{\omega t}{2}\right)^{2}}\left(\xi(t)^{2}+\eta(t)^{2}\right)+\frac{(\omega T-\sin (\omega T)) t}{8 \omega \sin \left(\frac{\omega T}{2}\right)^{2} T}\left(\varphi_{1}(T, 0)^{2}+\varphi_{2}(T, 0)^{2}\right) .
\end{aligned}
$$

In the last equality one has to substitute for $\varphi_{1}(t, 0)$ and $\varphi_{2}(t, 0)$ from (A.9), and for $\xi(t)$ and $\eta(t)$ from (40).

It is of importance to observe that the functions $F_{1}(t), F_{2}(t)$ and $\Phi(t)$ entering formula (41) are continuously differentiable. In addition, they are necessarily $T$ periodic. Furthermore, the operators $x$ and $p$ are infinitesimally small with respect to $H_{\omega}$. This is a well known fact which is also briefly recalled in the beginning of the Appendix. From equality (39) one can see that Dom $H_{F}=\operatorname{Dom} H_{\omega}$. Moreover, from the commutation relations (38) it follows that the unitary groups $\{\exp (\mathrm{i} s x) ; s \in \mathbb{R}\}$ and $\{\exp (\mathrm{i} s p) ; s \in \mathbb{R}\}$ preserve the domain Dom $H_{\omega}$. Hence one can differentiate $U_{F}(t)$ given in (41) on any vector $\psi \in$ Dom $H_{F}$. Computing $S_{F}(t)$ according to (16) one finds that

$$
S_{F}(t)=-\frac{F_{1}^{\prime}(t)}{\omega} p-F_{2}^{\prime}(t) x+\frac{F_{1}(t) F_{2}^{\prime}(t)}{\omega}-\Phi^{\prime}(t) .
$$


Consequently, $S_{F}(t)$ is infinitesimally small with respect to $H_{F}$ for any $t$. Thus all assumptions of Proposition 10 are fulfilled and one concludes that $\|H(t) U(t, 0) \psi\|$ is bounded in time for any $\psi \in \operatorname{Dom}(H(0))=\operatorname{Dom}\left(H_{\omega}\right)$.

From the explicit form of $H(t)$ and from the infinitesimal smallness of $x$ with respect to $H_{\omega}$ it follows that the quantity $\left\|H_{\omega} U(t, 0) \psi\right\|$ is bounded in time as well. Let us recall once more the consequences of this observation. Firstly, as stressed in [20, Proposition 4], since $F\left(H_{\omega}<R\right)$ is a finite rank projector for any $R>0$ it is true that all trajectories $\{U(t, 0) \psi ; t \in \mathbb{R}\}$ are precompact. Secondly, in virtue of Theorem 2.3 in [13], the monodromy operator $U(T, 0)$ has a pure point spectrum.

Finally, let us shortly discuss the resonant case $T=(2 \pi / \omega) N, N \in \mathbb{N}$. Using again formula (A.8) we have

$$
U(T, 0)=(-1)^{N} e^{-\mathrm{i} \psi(T, 0)} \exp \left(-\mathrm{i} \varphi_{1}(T, 0) x\right) \exp \left(\mathrm{i} \frac{\varphi_{2}(T, 0)}{\omega} p\right) .
$$

Notice that the unitary operator $e^{\mathrm{i} \alpha x} e^{\mathrm{i} \beta p}$, with $\alpha, \beta \in \mathbb{R}$, is either the identity if $\alpha=\beta=0$ or it has a purely absolutely continuous spectrum. For example, if $\beta \neq 0$ then we have the commutation relation

$$
e^{\mathrm{i} \alpha x} e^{\mathrm{i} \beta p}=\exp \left(-\mathrm{i} \frac{\alpha}{2 \beta} x^{2}\right) \exp \left(\mathrm{i} \beta p-\frac{\mathrm{i}}{2} \alpha \beta\right) \exp \left(\mathrm{i} \frac{\alpha}{2 \beta} x^{2}\right) .
$$

Hence the spectrum of $e^{\mathrm{i} \alpha x} e^{\mathrm{i} \beta p}$ coincides with that of $e^{-\mathrm{i} \alpha \beta / 2} e^{\mathrm{i} \beta p}$. In the case $\alpha \neq 0$ one can argue in a similar way. Thus when applying this observation to (42) we have to distinguish the case $\varphi_{1}(T, 0)=\varphi_{2}(T, 0)=0$. Recalling defining relations (A.9) we denote by

$$
f_{k}=\frac{1}{T} \int_{0}^{T} \exp \left(-\mathrm{i} \frac{2 \pi}{T} k t\right) f(t) \mathrm{d} t, \quad k \in \mathbb{Z},
$$

the Fourier coefficients of $f(t)$. We conclude that if $f_{-N}=f_{N}=0$ then the monodromy operator $U(T, 0)$, with $T=2 \pi N / \omega$, is a multiple of the identity. If $\left|f_{-N}\right|+\left|f_{N}\right|>0$ then $U(T, 0)$ has a purely absolutely continuous spectrum. This in turn implies that, in the latter case, the quantity $\|H(t) U(t, 0) \psi\|$ cannot happen to be bounded in time for all $\psi \in \operatorname{Dom} H_{\omega}$.

\section{An application of the quantum KAM method}

The quantum KAM method was originally proposed by Bellissard [4] and it has been later reconsidered and in some respects improved several times, see for example 9 , 8, 12, 2, 11. When discussing an application of the quantum KAM method to our problem we shall stick to the presentation given in [11] but the notation will be partially modified. A particularity of the method is that the frequency $\omega=2 \pi / T$ should be considered as a parameter. Usually the method is used to show that for a large subset of so called non-resonant frequencies the spectrum of the Floquet Hamiltonian is pure point. Here we would like to point out, following some ideas from [1], that the method 
provides a more detailed information which can be used to reveal the structure of the propagator.

Let us first recall the main theorem from [11]. Let $H_{0}$ be a self-adjoint operator in $\mathscr{H}$ with a discrete spectrum, $\operatorname{Spec}\left(H_{0}\right)=\left\{h_{m}\right\}_{m=1}^{\infty}$, and such that the multiplicities $M_{m}=\operatorname{dim} \operatorname{Ker}\left(H_{0}-h_{m}\right)$ are finite. Suppose also that

$$
\Delta_{0}=\inf _{m \neq n}\left|h_{m}-h_{n}\right|>0
$$

Furthermore, let $V(t)$ be a $2 \pi$-periodic uniformly bounded operator-valued function defined on $\mathbb{R}$ and with values in $\mathscr{B}(\mathscr{H})$. Set

$$
V_{k n m}=\frac{1}{2 \pi} \int_{0}^{2 \pi} e^{-\mathrm{i} k t} Q_{n} V(t) Q_{m} \mathrm{~d} t
$$

where $Q_{n}$ is the orthogonal projector onto $\operatorname{Ker}\left(H_{0}-h_{n}\right)$. As already mentioned, the frequency $\omega=2 \pi / T, T>0$, is regarded as a parameter. Set $\mathscr{K}=L^{2}([0, T], \mathscr{H}, \mathrm{d} t)$ and let $V \in \mathscr{B}(\mathscr{K})$ be the operator acting via multiplication by $V(\omega t),(V f)(t)=$ $V(\omega t) f(t)$. Let $K_{0}$ be the closure of $-\mathrm{i} \partial_{t} \otimes 1+1 \otimes H_{0}$.

Theorem 27. Fix $J>0$ and set $\Omega_{0}=\left[\frac{8}{9} J, \frac{9}{8} J\right]$. Assume that there exists $\sigma>0$ such that

$$
\sum_{\substack{m, n \in \mathbb{N} \\ h_{m}-h_{n}>J / 2}} \frac{M_{m} M_{n}}{\left(h_{m}-h_{n}\right)^{\sigma}}<\infty .
$$

Then for every $r>\sigma+\frac{1}{2}$ there exist positive constants (depending on $\sigma, r, \Delta_{0}$ and $J$ but independent of $V), \epsilon_{\star}$ and $\delta_{\star}$, with the property:

if

$$
\epsilon_{V}:=\sup _{n \in \mathbb{N}} \sum_{m \in \mathbb{N}} \sum_{k \in \mathbb{Z}}(1+|k|)^{r}\left\|V_{k n m}\right\|<\epsilon_{\star}
$$

then there exists a measurable subset $\Omega_{\infty} \subset \Omega_{0}$ such that

$$
\left|\Omega_{\infty}\right| \geq\left|\Omega_{0}\right|-\delta_{\star} \epsilon_{V}
$$

(here $\left|\Omega_{*}\right|$ stands for the Lebesgue measure of $\Omega_{*}$ ) and the operator $K_{0}+V$ has a pure point spectrum for all $\omega \in \Omega_{\infty}$.

The proof of Theorem 27 is somewhat lengthy and tedious because one has to eliminate the resonant frequencies. The basic idea is, however, rather simple and is based on an iterative procedure as described in the following proposition. It is formulated even on the level of Banach spaces but afterwards we shall again work with Hilbert spaces. Let $\Phi(x)$ be the analytic function defined by

$$
\Phi(x)=\frac{1}{x}\left(e^{x}-\frac{e^{x}-1}{x}\right) .
$$


Proposition 28. Assume that $\mathscr{K}$ is a Banach space, $K_{0}$ is a closed operator in $\mathscr{K}$, $V \in \mathscr{B}(\mathscr{K})$ and $D \in \mathscr{B}(\mathscr{B}(\mathscr{K}))$. Assume further that $V=\lim V_{s}$ in $\mathscr{B}(\mathscr{K})$ where $\left\{V_{s}\right\}_{s=0}^{\infty}$ is a sequence of bounded operators in $\mathscr{K}$. If there exist sequences $\left\{A_{s}\right\}_{s=0}^{\infty}$ and $\left\{G_{s}\right\}_{s=0}^{\infty}, A_{s}, G_{s} \in \mathscr{B}(\mathscr{K})$, fulfilling the following recurrence relations for all $s \in \mathbb{Z}_{+}$:

$$
\begin{aligned}
G_{0}= & V_{0}, \\
G_{s+1}= & G_{s}+\exp \left(\operatorname{ad}_{A_{s}}\right) \ldots \exp \left(\operatorname{ad}_{A_{0}}\right)\left(V_{s+1}-V_{s}\right) \\
& +\operatorname{ad}_{A_{s}} \Phi\left(\operatorname{ad}_{A_{s}}\right)(1-D)\left(G_{s}-G_{s-1}\right),
\end{aligned}
$$

$A_{s} \operatorname{Dom}\left(K_{0}\right) \subset \operatorname{Dom}\left(K_{0}\right)$,

$$
\begin{aligned}
{\left[A_{0}, K_{0}+D\left(G_{0}\right)\right] } & =-(1-D)\left(G_{0}\right), \\
{\left[A_{s+1}, K_{0}+D\left(G_{s+1}\right)\right] } & =-(1-D)\left(G_{s+1}-G_{s}\right),
\end{aligned}
$$

and such that $\sum_{s=0}^{\infty}\left\|A_{s}\right\|<\infty$ and the limit $\lim G_{s}=G_{\infty}$ exists in $\mathscr{B}(\mathscr{K})$ then there exists $W \in \mathscr{B}(\mathscr{K})$ such that $W^{-1} \in \mathscr{B}(\mathscr{K})$ and

$$
W\left(K_{0}+V\right) W^{-1}=K_{0}+D\left(G_{\infty}\right) .
$$

Here, as usual, ad means the adjoint action, $\operatorname{ad}_{A} X=[A, X]$ and $\exp \left(\operatorname{ad}_{A}\right) X=$ $e^{A} X e^{-A}$. For $s=0$ in (45) we set $G_{-1}=0$. The proof of Proposition 28 is immediate. If we set

$$
W_{s}=e^{A_{s-1}} \ldots e^{A_{0}}, W_{s}^{-1}=e^{-A_{0}} \ldots e^{-A_{s-1}},
$$

then the recurrence relations (45), (46) exactly mean that

$$
\forall s \in \mathbb{Z}_{+}, W_{s}\left(K_{0}+V_{s}\right) W_{s}^{-1}=K_{0}+D\left(G_{s}\right)+(1-D)\left(G_{s}-G_{s-1}\right) .
$$

Now it suffices to send $s$ to infinity.

In the applications of Proposition 28, and this is also the case for Theorem 27, $\mathscr{K}$ is a separable Hilbert space, $K_{0}=K_{0}^{*}, V=V^{*}$, the spectrum of $K_{0}$ is pure point and $D(X)$ is the diagonal part of a bounded operator $X$ with respect to the spectral decomposition of $K_{0}$. Then $G_{\infty}^{*}=G_{\infty}, D\left(G_{\infty}\right)^{*}=D\left(G_{\infty}\right)$ and $W^{*}=W^{-1}$. The operator $K_{0}+D\left(G_{\infty}\right)$ has obviously a pure point spectrum and relation (47) implies that the same is true for $K_{0}+V$.

Let us note that technically the basic problem of the entire method is the commutator equation (46) whose solution is complicated by the fact that, generically, the eigenvalues of $K_{0}$ are dense in $\mathbb{R}$. This leads to the famous problem of small denominators in this context.

There is another feature concerning the application of the recursive procedure (45) and (46) in the proof of Theorem [27. Let $M \in \mathscr{B}(\mathscr{K})$ be the multiplication operator defined by the relation

$$
\forall f \in \mathscr{K},(M f)(t)=e^{\mathrm{i} \omega t} f(t) .
$$

Since $V \in \mathscr{B}(\mathscr{K})$ is a multiplication operator it commutes with $M$. Also the sequence $\left\{V_{s}\right\}$ is chosen in such a way that $M$ commutes with all $V_{s}$. Furthermore, the eigenvalues of $K_{0}$ are $k \omega+h_{m}, k \in \mathbb{Z}$ and $m \in \mathbb{N}$, and so they are linear in 
$k$. Using these facts it is readily seen from the recursive relations that $M$ commutes with both $A_{s}$ and $G_{s}$ for all $s$. Then necessarily $M$ commutes with $G_{\infty}$ and $W$ as well. This implies that there exists a bounded Hermitian operator $G$ on $\mathscr{H}$ such that $G \operatorname{Dom}\left(H_{0}\right) \subset \operatorname{Dom}\left(H_{0}\right)$,

$$
\left[H_{0}, G\right]=0 \text { and }\left(D\left(G_{\infty}\right) f\right)(t)=G f(t), \quad \forall f \in \mathscr{K}, \text { a.a. } t \in \mathbb{R}
$$

and there exists a $T$-periodic operator-valued function $t \mapsto W(t)$ with values in unitary operators on $\mathscr{H}$ such that equality (47) is satisfied with

$$
(W f)(t)=W(t) f(t), \quad \forall f \in \mathscr{K}, \text { a.a. } t \in \mathbb{R} .
$$

Moreover, an information about the regularity of $W$ is also available. More precisely, one knows that

$$
\sup _{n \in \mathbb{N}} \sum_{m \in \mathbb{N}} \sum_{k \in \mathbb{Z}}\left\|W_{k n m}\right\|<\infty
$$

where again

$$
W_{k n m}=\frac{1}{T} \int_{0}^{T} e^{-\mathrm{i} k \omega t} Q_{n} W(t) Q_{m} \mathrm{~d} t .
$$

Particularly, the operator-valued function $W(t)$ is continuous even in the operator norm. Equality (47) can be rewritten in terms of propagators. It exactly means that

$$
\forall t, s \in \mathbb{R}, U(t, s)=W(t)^{*} e^{-\mathrm{i}(t-s)\left(H_{0}+G\right)} W(s) .
$$

By a closer look at the proof of Theorem 27 one finds that the result can be partially improved. In the course of the proof one constructs a directed sequence of Banach spaces $\left\{\mathfrak{X}_{s}\right\}$,

$$
\mathfrak{X}_{s} \subset L^{\infty}\left(\Omega_{s} \times \mathbb{Z} \times \mathbb{N} \times \mathbb{N}, \sum_{n \in \mathbb{N}} \sum_{m \in \mathbb{N}}^{\oplus} \mathscr{B}\left(\mathcal{H}_{m}, \mathcal{H}_{n}\right)\right)
$$

with the norms

$$
\|X\|_{s}=\sup _{\substack{\omega, \omega^{\prime} \in \Omega_{s} \\ \omega \neq \omega^{\prime}}} \sup _{n \in \mathbb{N}} \sum_{m \in \mathbb{N}} \sum_{k \in \mathbb{Z}}\left(\left\|X_{k n m}(\omega)\right\|+\varphi_{s}\left\|\widetilde{\partial} X_{k n m}\left(\omega, \omega^{\prime}\right)\right\|\right) e^{|k| / \mathcal{E}_{s}}
$$

where $X=\left\{X_{k n m}(\omega)\right\} \in \mathfrak{X}_{s}$, i.e., $X_{k n m}(\omega) \in \mathscr{B}\left(\mathcal{H}_{m}, \mathcal{H}_{n}\right)$ for all $\omega \in \Omega_{s}$ and $(k, n, m) \in \mathbb{Z} \times \mathbb{N} \times \mathbb{N}$. Here $\mathcal{H}_{m}:=\operatorname{Ker}\left(H_{0}-h_{m}\right)=\operatorname{Ran} Q_{m},\left\{\Omega_{s}\right\}$ is a decreasing sequence of subsets of the interval $\Omega_{0},\left\{\varphi_{s}\right\}$ and $\left\{\mathcal{E}_{s}\right\}$ are respectively decreasing and strictly increasing sequences of positive numbers such that $\lim \varphi_{s}=0,1 \leq \mathcal{E}_{s}$ and $\lim \mathcal{E}_{s}=+\infty$. The symbol $\widetilde{\partial}$ designates the discrete derivative in $\omega$,

$$
\widetilde{\partial} X\left(\omega, \omega^{\prime}\right)=\frac{X(\omega)-X\left(\omega^{\prime}\right)}{\omega-\omega^{\prime}} .
$$


For $\omega \in \Omega_{\infty}=\bigcap \Omega_{s}$ fixed one applies the limit procedure $s \rightarrow \infty$ and arrives at equality (47) with the objects $G_{\infty}$ and $W$ belonging to the Banach space

$$
\mathfrak{X}_{\infty} \subset L^{\infty}\left(\mathbb{Z} \times \mathbb{N} \times \mathbb{N}, \sum_{n \in \mathbb{N}} \sum_{m \in \mathbb{N}}^{\oplus} \mathscr{B}\left(\mathcal{H}_{m}, \mathcal{H}_{n}\right)\right)
$$

where the norm is defined by

$$
\|X\|_{\infty}=\sup _{n \in \mathbb{N}} \sum_{m \in \mathbb{N}} \sum_{k \in \mathbb{Z}}\left\|X_{k n m}(\omega)\right\| .
$$

This is also how one obtains the information about the regularity of $W$ expressed in (49).

The announced improvement consists in modifying the norms (51) by an additional weight $(1+|k|)^{\nu}$ where $\nu$ should be chosen in the range

$$
0 \leq \nu<r-\sigma-\frac{1}{2}
$$

Recall that $r$ determines the regularity of $V$ in (44), $\sigma$ comes from the "gap condition" (43) and one requires that $r>\sigma+\frac{1}{2}$. The modified norm reads

$$
\|X\|_{s}=\sup _{\substack{\omega, \omega^{\prime} \in \Omega_{s} \\ \omega \neq \omega^{\prime}}} \sup _{n \in \mathbb{N}} \sum_{m \in \mathbb{N}} \sum_{k \in \mathbb{Z}}(1+|k|)^{\nu}\left(\left\|X_{k n m}(\omega)\right\|+\varphi_{s}\left\|\widetilde{\partial} X_{k n m}\left(\omega, \omega^{\prime}\right)\right\|\right) e^{|k| / \mathcal{E}_{s}}
$$

and the limit procedure results in a norm in $\mathfrak{X}_{\infty}$,

$$
\|X\|_{\infty}=\sup _{n \in \mathbb{N}} \sum_{m \in \mathbb{N}} \sum_{k \in \mathbb{Z}}(1+|k|)^{\nu}\left\|X_{k n m}(\omega)\right\| .
$$

Let us note that restriction (52) comes from the lower estimate of Lebesgue measure of the set $\Omega_{\infty}$ (see relation (77) in [1] and the derivation preceding it where one has to replace $r$ by $r-\nu$ if using the modified norm (53) $)$. After this modification, Theorem 27 is valid exactly in the same formulation as before, its proof requires no additional changes, only the constants $\epsilon_{\star}$ and $\delta_{\star}$ should be modified correspondingly.

The interest of the modification is that we get a better information about the regularity of $W$. Namely, for $\omega \in \Omega_{\infty}$ (the set of non-resonant frequencies) $W$ is regular in the sense that $\|W\|_{\infty}<\infty$ with the norm given by (54). In particular, if $r>\sigma+\frac{3}{2}$ then one can choose $\nu \geq 1$. In that case the property $\|W\|_{\infty}<\infty$ implies that $W(t)$ belongs to the class $C^{1}$ in the operator norm and $\sup _{t}\left\|\partial_{t} W(t)\right\|<\infty$.

This discussion shows that Theorem 27 can be reformulated in the following way.

Theorem 29. Under the same assumptions as in Theorem 27 suppose that $r>\sigma+\frac{3}{2}$. Then there exist positive constants (independent of $V$ ), $\epsilon_{\star}$ and $\delta_{\star}$, with the property:

if $\epsilon_{V}<\epsilon_{\star}$ then there exists a measurable subset $\Omega_{\infty} \subset \Omega_{0}$ such that $\left|\Omega_{\infty}\right| \geq$ $\left|\Omega_{0}\right|-\delta_{\star} \epsilon_{V}$, and for every $\omega \in \Omega_{\infty}$ there exist a bounded Hermitian operator $G$ commuting with $H_{0}$ and a T-periodic function $W(t)$ with values in unitary operators and belonging to the class $C^{1}$ in the operator norm such that the propagator obeys equality (50). 
From relation (50) it follows that the propagator admits a Floquet decomposition (15) with

$$
H_{F}=W(0)^{*}\left(H_{0}+G\right) W(0), U_{F}(t)=W(t)^{*} W(0) .
$$

Moreover, formula (16) implies that

$$
S_{F}(t)=-\mathrm{i} W(0)^{*}\left(\partial_{t} W(t)\right) W(t)^{*} W(0) .
$$

In particular, if $W(t)$ is known to be $C^{1}$ in the operator norm then the Floquet decomposition is continuously differentiable in the strong sense and, consequently, the assumptions both of Proposition 10 and Proposition 12 are satisfied. These arguments prove the following theorem.

Theorem 30. Under the same assumptions as in Theorem 27 suppose that $r>\sigma+\frac{3}{2}$. Then there exist positive constants (independent of $V$ ), $\epsilon_{\star}$ and $\delta_{\star}$, with the property:

if $\epsilon_{V}<\epsilon_{\star}$ (with $\epsilon_{V}$ defined in (44)) then there exists a measurable subset $\Omega_{\infty} \subset \Omega_{0}$ such that $\left|\Omega_{\infty}\right| \geq\left|\Omega_{0}\right|-\delta_{\star} \epsilon_{V}$ and for every $\omega \in \Omega_{\infty}$ the energy of the quantum system described by the time-dependent Hamiltonian $H_{0}+V(\omega t)$ is bounded uniformly in time. More precisely,

$$
\forall \psi \in \operatorname{Dom}(H(0)), \sup _{t \in \mathbb{R}}\left\|\left(H_{0}+V(\omega t)\right) U(t, 0) \psi\right\|<\infty .
$$

Moreover, there exists a constant $c \geq 0$ such that for any couple of intervals $\Delta_{1}, \Delta_{2} \subset$ $\mathbb{R}$ fulfilling $\operatorname{dist}\left(\Delta_{1}, \Delta_{2}\right)>0$ it holds true that

$$
\sup _{s, t \in \mathbb{R}}\left\|P\left(t, \Delta_{1}\right) U(t, s) P\left(s, \Delta_{2}\right)\right\| \leq \frac{c}{\operatorname{dist}\left(\Delta_{1}, \Delta_{2}\right)}
$$

where $P(t, \cdot)$ is the spectral measure of $H(t)$. In particular, if $E_{n}(t)$ and $E_{m}(s)$ are two distinct eigenvalues of $H(t)$ and $H(s)$, respectively, and $P_{n}(t)$ and $P_{m}(s)$ are the corresponding orthogonal projectors then

$$
\left\|P_{n}(t) U(t, s) P_{m}(s)\right\| \leq \frac{c}{\left|E_{n}(t)-E_{m}(s)\right|} .
$$

\section{Acknowledgments}

P. Š. wishes to acknowledge gratefully the support from the grant No. 201/05/0857 of Grant Agency of the Czech Republic. 


\section{Appendix. The propagator for the time-dependent harmonic oscillator}

Let $H_{\omega}=(1 / 2)\left(p^{2}+\omega^{2} x^{2}\right)$, with $p=-\mathrm{i} \partial_{x}$, be the Hamiltonian of the harmonic oscillator in $\mathscr{H}=L^{2}(\mathbb{R}, \mathrm{d} x)$. This is a known fact that the operators $x$ and $p$ are relatively bounded with respect to $H_{\omega}$ with the relative bound zero. One can see it also directly from the following inequality which is easy to verify on the Schwarz space $\mathscr{S}$ :

$$
2 \omega^{2} x^{2} \leq \varepsilon^{-2}+\varepsilon^{2} \omega^{4} x^{4}+\varepsilon^{2}\left(p^{4}+2 \omega^{2} p x^{2} p\right)=\varepsilon^{-2}+2 \varepsilon^{2} \omega^{2}+4 \varepsilon^{2} H_{\omega}^{2} .
$$

Hence it is true that

$$
\|x \psi\|^{2} \leq\left(\varepsilon^{2}+\frac{1}{2 \omega^{2} \varepsilon^{2}}\right)\|\psi\|^{2}+\frac{2 \varepsilon^{2}}{\omega^{2}}\left\|H_{\omega} \psi\right\|^{2}
$$

for all $\psi \in \mathscr{S}$ and consequently for all $\psi \in \operatorname{Dom} H_{\omega}$. A bound for the operator $p$ can be derived analogously. It follows that for any $\alpha, \beta \in \mathbb{R}$, the domain $\operatorname{Dom}\left(H_{\omega}+\alpha x+\beta p\right)$ coincides with Dom $H_{\omega}$.

For $\varphi, \psi \in \operatorname{Dom}\left(H_{\omega}\right)$ set

$$
\mathbf{x}(t)=\left\langle e^{-\mathrm{i} t H_{\omega}} \varphi, x e^{-\mathrm{i} t H_{\omega}} \psi\right\rangle, \mathbf{p}(t)=\left\langle e^{-\mathrm{i} t H_{\omega}} \varphi, p e^{-\mathrm{i} t H_{\omega}} \psi\right\rangle .
$$

As a standard exercise one derives, by differentiating and using the canonical commutation relation, that the quantities $\mathbf{x}(t)$ and $\mathbf{p}(t)$ obey the classical evolution equations, i.e., $\dot{\mathbf{x}}(t)=\mathbf{p}(t), \dot{\mathbf{p}}(t)=-\omega^{2} \mathbf{x}(t)$. It follows that for all $\psi \in \operatorname{Dom}\left(H_{\omega}\right)$,

$$
\begin{aligned}
e^{\mathrm{i} t H_{\omega}} x e^{-\mathrm{i} t H_{\omega}} \psi & =\cos (\omega t) x \psi+\frac{1}{\omega} \sin (\omega t) p \psi, \\
e^{\mathrm{i} t H_{\omega}} p e^{-\mathrm{i} t H_{\omega}} \psi & =-\omega \sin (\omega t) x \psi+\cos (\omega t) p \psi .
\end{aligned}
$$

Lemma A.1. For $\mu, \nu, t \in \mathbb{R}$ it holds

$$
\exp \left(-\mathrm{i} t H_{\omega}+\mathrm{i} \frac{\mu}{\omega} p+\mathrm{i} \nu x\right)=e^{-\mathrm{i} \phi} \exp \left(\mathrm{i} \frac{\xi}{\omega} p\right) \exp (\mathrm{i} \eta x) \exp \left(-\mathrm{i} t H_{\omega}\right)
$$

where

$$
\begin{aligned}
& \xi=\frac{2 \sin \left(\frac{\omega t}{2}\right)}{\omega t}\left(\cos \left(\frac{\omega t}{2}\right) \mu-\sin \left(\frac{\omega t}{2}\right) \nu\right), \\
& \eta=\frac{2 \sin \left(\frac{\omega t}{2}\right)}{\omega t}\left(\sin \left(\frac{\omega t}{2}\right) \mu+\cos \left(\frac{\omega t}{2}\right) \nu\right),
\end{aligned}
$$

and

$$
\begin{aligned}
\phi= & -\frac{1}{4 \omega^{3} t^{2}}\left((2 \omega t-4 \sin (\omega t)+\sin (2 \omega t)) \mu^{2}+(2-4 \cos (\omega t)+2 \cos (2 \omega t)) \mu \nu\right. \\
& \left.+(2 \omega t-\sin (2 \omega t)) \nu^{2}\right)
\end{aligned}
$$


Proof. Set

$$
W_{1}=\exp \left(\mathrm{i} \frac{\xi}{\omega} p\right) \exp (\mathrm{i} \eta x) \exp \left(-\mathrm{i} t H_{\omega}\right), W_{2}=\exp \left(-\mathrm{i} t H_{\omega}+\mathrm{i} \frac{\mu}{\omega} p+\mathrm{i} \nu x\right) .
$$

Clearly, both $W_{1}$ and $W_{2}$ leave the domain of $H_{\omega}$ invariant. Using (A.1) one finds that for all $\psi \in \operatorname{Dom}\left(H_{\omega}\right)$ it holds

$$
\begin{aligned}
& W_{1}^{-1} x W_{1} \psi=\cos (\omega t) x \psi+\frac{1}{\omega} \sin (\omega t) p \psi-\frac{\xi}{\omega} \psi \\
& W_{1}^{-1} p W_{1} \psi=-\omega \sin (\omega t) x \psi+\cos (\omega t) p \psi+\eta \psi
\end{aligned}
$$

On the other hand,

$$
-\mathrm{i} t H_{\omega}+\mathrm{i} \frac{\mu}{\omega} p+\mathrm{i} \nu x=-\mathrm{i} \frac{t}{2}\left(\widetilde{p}^{2}+\omega^{2} \widetilde{x}^{2}\right)+\mathrm{i} \frac{\mu^{2}+\nu^{2}}{2 \omega^{2} t}
$$

where

$$
\widetilde{x}=x-\frac{\nu}{\omega^{2} t}, \widetilde{p}=p-\frac{\mu}{\omega t} .
$$

Since $\widetilde{p}$ and $\widetilde{x}$ also obey the canonical commutation relation we have, analogously to (A.1),

$$
\begin{aligned}
W_{2}^{-1} \widetilde{x} W_{2} \psi & =\cos (\omega t) \widetilde{x} \psi+\frac{1}{\omega} \sin (\omega t) \widetilde{p} \psi \\
W_{2}^{-1} \widetilde{p} W_{2} \psi & =-\omega \sin (\omega t) \widetilde{x} \psi+\cos (\omega t) \widetilde{p} \psi
\end{aligned}
$$

for all $\psi \in \operatorname{Dom}\left(H_{\omega}\right)$. This can be rewritten as

$$
\begin{aligned}
& W_{2}^{-1} x W_{2} \psi=\cos (\omega t) x \psi+\frac{1}{\omega} \sin (\omega t) p \psi+\frac{1}{\omega^{2} t}(\nu-\nu \cos (\omega t)-\mu \sin (\omega t)) \psi \\
& W_{2}^{-1} p W_{2} \psi=-\omega \sin (\omega t) x \psi+\cos (\omega t) p \psi+\frac{1}{\omega t}(\mu+\nu \sin (\omega t)-\mu \cos (\omega t)) \psi
\end{aligned}
$$

Comparing (A.5) to (A.6) one finds that for all $\psi \in \operatorname{Dom}\left(H_{\omega}\right)$ it holds $W_{1}^{-1} x W_{1} \psi=$ $W_{2}^{-1} x W_{2} \psi$ and $W_{1}^{-1} p W_{1} \psi=W_{2}^{-1} p W_{2} \psi$ provided

$$
\xi=-\frac{\nu}{\omega t}(1-\cos (\omega t))+\frac{\mu}{\omega t} \sin (\omega t), \eta=\frac{\nu}{\omega t} \sin (\omega t)+\frac{\mu}{\omega t}(1-\cos (\omega t))
$$

(which is nothing but (A.3)). Hence $W=W_{2} W_{1}^{-1}$ fulfills $W^{-1} x W \psi=x \psi$ and $W^{-1} p W \psi=p \psi$. Since $\operatorname{Dom}\left(H_{\omega}\right)$ is a core both for $x$ and $p$ this implies that $W^{-1} x W=$ $x$ and $W^{-1} p W=p$. If follows that $W$ is a multiple of the unity, i.e., $W_{2}=e^{-\mathrm{i} \phi} W_{1}$ for some $\phi \in \mathbb{R}$.

It remains to determine $\phi$. To this end it suffices to take the mean value of the corresponding operators at the ground state of $H_{\omega}$ which is $\psi_{0}(x)=\exp \left(-\omega x^{2} / 2\right)$ (unnormalized). Writing

$$
W_{2}=\exp \left(\mathrm{i} \frac{\mu^{2}+\nu^{2}}{2 \omega^{2} t}\right) \exp \left(-\mathrm{i} \frac{\nu}{\omega^{2} t} p\right) \exp \left(\mathrm{i} \frac{\mu}{\omega t} x\right) e^{-\mathrm{i} t H_{\omega}} \exp \left(-\mathrm{i} \frac{\mu}{\omega t} x\right) \exp \left(\mathrm{i} \frac{\nu}{\omega^{2} t} p\right)
$$


the equality $W_{2}=e^{-\mathrm{i} \phi} W_{1}$ becomes

$$
\begin{aligned}
& \exp \left(\mathrm{i} \frac{\mu^{2}+\nu^{2}}{2 \omega^{2} t}\right) e^{-\mathrm{i} t H_{\omega}} \exp \left(-\mathrm{i} \frac{\mu}{\omega t} x\right) \exp \left(\mathrm{i} \frac{\nu}{\omega^{2} t} p\right) \\
& =\exp \left(-\mathrm{i} \phi+\mathrm{i}\left(\frac{\nu}{\omega^{2} t}+\frac{\xi}{\omega}\right) \eta\right) \exp \left(\mathrm{i}\left(\eta-\frac{\mu}{\omega t}\right) x\right) \exp \left(\mathrm{i}\left(\frac{\xi}{\omega}+\frac{\nu}{\omega^{2} t}\right) p\right) e^{-\mathrm{i} t H_{\omega}} .
\end{aligned}
$$

The mean value at $\psi_{0}$ of the both sides of the last equality then yields

$$
\begin{aligned}
& \exp \left(\mathrm{i}\left(\phi+\frac{\mu^{2}+\nu^{2}}{2 \omega^{2} t}-\left(\frac{\nu}{\omega^{2} t}+\frac{\xi}{\omega}\right) \eta\right)\right)\left\langle\psi_{0}(x), \exp \left(-\mathrm{i} \frac{\mu}{\omega t} x\right) \psi_{0}\left(x+\frac{\nu}{\omega^{2} t}\right)\right\rangle \\
& =\left\langle\psi_{0}(x), \exp \left(\mathrm{i}\left(\eta-\frac{\mu}{\omega t}\right) x\right) \psi_{0}\left(x+\frac{\xi}{\omega}+\frac{\nu}{\omega^{2} t}\right)\right\rangle .
\end{aligned}
$$

A straightforward computation then leads to the value (A.4).

Conversely, one can express $\mu$ and $\nu$ in terms of $\xi, \eta$ and $t$ provided $t$ is sufficiently small. In other words, one can read equality (A.2) from the right to the left. The restriction on smallness of $t$ should not be considered as surprising since the reversed equality is in fact an application of the Baker-Campbell-Haussdorf formula which is known to be guaranteed only locally.

Lemma A.2. For $\xi, \eta, t \in \mathbb{R},|t|<2 \pi / \omega$, it holds

$$
\exp \left(\mathrm{i} \frac{\xi}{\omega} p\right) \exp (\mathrm{i} \eta x) \exp \left(-\mathrm{i} t H_{\omega}\right)=e^{\mathrm{i} \phi} \exp \left(-\mathrm{i} t H_{\omega}+\mathrm{i} \frac{\mu}{\omega} p+\mathrm{i} \nu x\right)
$$

where

$$
\mu=\frac{\omega t}{2}\left(\cot \left(\frac{\omega t}{2}\right) \xi+\eta\right), \nu=\frac{\omega t}{2}\left(-\xi+\cot \left(\frac{\omega t}{2}\right) \eta\right)
$$

and

$$
\phi=\frac{1}{2 \omega} \xi \eta-\frac{\omega t-\sin (\omega t)}{8 \omega \sin \left(\frac{\omega t}{2}\right)^{2}}\left(\xi^{2}+\eta^{2}\right) .
$$

Set

$$
H(t)=H_{\omega}+f(t) x
$$

where the function $f(t)$ is supposed to be continuous and $T$ periodic. In [13] a formula has been derived for the propagator corresponding to the Hamiltonian $H(t)$ :

$$
U(t, s)=\exp \left(-\mathrm{i} \varphi_{1}(t, s) x\right) \exp \left(\mathrm{i} \frac{\varphi_{2}(t, s)}{\omega} p\right) \exp \left(-\mathrm{i}(t-s) H_{\omega}-\mathrm{i} \psi(t, s)\right)
$$

where

$$
\begin{aligned}
\varphi_{1}(t, s) & =\int_{s}^{t} \cos (\omega(t-u)) f(u) \mathrm{d} u \\
\varphi_{2}(t, s) & =\int_{s}^{t} \sin (\omega(t-u)) f(u) \mathrm{d} u
\end{aligned}
$$


and

$$
\psi(t, s)=\frac{1}{2} \int_{s}^{t}\left(\varphi_{1}(v, s)^{2}-\varphi_{2}(v, s)^{2}\right) \mathrm{d} v
$$

(be aware of a sign error in the definition of $\psi(t, s)$ in [13]). Our goal is to transform formula (A.8) due to Enss and Veselic into another one expressing the propagator as a single exponential function of an operator.

Proposition A.3. For $t, s \in \mathbb{R},(t-s) \notin(2 \pi / \omega) \mathbb{Z}$, set

$$
\left.N=\left[\frac{\omega(t-s)}{2 \pi}\right] \in \mathbb{Z}, \Delta=\frac{2 \pi}{\omega}\left\{\frac{\omega(t-s)}{2 \pi}\right\} \in\right] 0, \frac{2 \pi}{\omega}[
$$

(where $[x]$ and $\{x\}$ are respectively the integer part and the fractional part of $x$ ). Then it holds

$$
U(t, s)=(-1)^{N} \exp \left(-\mathrm{i} \Delta H_{\omega}+\mathrm{i} \frac{\mu(t, s)}{\omega} p+\mathrm{i} \nu(t, s) x+\mathrm{i} \sigma(t, s)\right)
$$

where

$$
\begin{aligned}
& \mu(t, s)=\frac{\omega \Delta}{2 \sin \left(\frac{\omega(t-s)}{2}\right)} \int_{s}^{t} \sin \left(\omega\left(\frac{t+s}{2}-u\right)\right) f(u) \mathrm{d} u \\
& \nu(t, s)=-\frac{\omega \Delta}{2 \sin \left(\frac{\omega(t-s)}{2}\right)} \int_{s}^{t} \cos \left(\omega\left(\frac{t+s}{2}-u\right)\right) f(u) \mathrm{d} u
\end{aligned}
$$

and

$$
\begin{aligned}
\sigma(t, s)= & -\frac{1}{2} \int_{s}^{t}\left(\varphi_{1}(v, s)^{2}-\varphi_{2}(v, s)^{2}\right) \mathrm{d} v+\frac{1}{2 \omega} \varphi_{1}(t, s) \varphi_{2}(t, s) \\
& -\frac{\omega \Delta-\sin (\omega(t-s))}{8 \omega \sin \left(\frac{\omega(t-s)}{2}\right)^{2}}\left(\varphi_{1}(t, s)^{2}+\varphi_{2}(t, s)^{2}\right) .
\end{aligned}
$$

Proof. We have $t-s=(2 \pi / \omega) N+\Delta$. Since the spectrum of $H_{\omega}$ equals $(\omega / 2)+\omega \mathbb{Z}_{+}$ it holds

$$
\exp \left(-\mathrm{i} \frac{2 \pi}{\omega} N H_{\omega}\right)=(-1)^{N}
$$

Combining (A.8) with Lemma A.2 we get

$$
\begin{aligned}
U(t, s) & =(-1)^{N} \exp \left(-\mathrm{i} \varphi_{1}(t, s) x\right) \exp \left(\mathrm{i} \frac{\varphi_{2}(t, s)}{\omega} p\right) \exp \left(-\mathrm{i} \Delta H_{\omega}-\mathrm{i} \psi(t, s)\right) \\
& =(-1)^{N} e^{\mathrm{i} \phi(t, s)} \exp \left(-\mathrm{i} \Delta H_{\omega}+\mathrm{i} \frac{\mu(t, s)}{\omega} p+\mathrm{i} \nu(t, s) x\right)
\end{aligned}
$$


where

$$
\begin{aligned}
& \mu(t, s)=\frac{\omega \Delta}{2}\left(\cot \left(\frac{\omega \Delta}{2}\right) \varphi_{2}(t, s)-\varphi_{1}(t, s)\right), \\
& \nu(t, s)=\frac{\omega \Delta}{2}\left(\varphi_{2}(t, s)+\cot \left(\frac{\omega \Delta}{2}\right) \varphi_{1}(t, s)\right),
\end{aligned}
$$

and

$$
\phi(t, s)=\frac{1}{2 \omega} \varphi_{1}(t, s) \varphi_{2}(t, s)-\frac{\omega \Delta-\sin (\omega \Delta)}{8 \omega \sin \left(\frac{\omega \Delta}{2}\right)^{2}}\left(\varphi_{1}(t, s)^{2}+\varphi_{2}(t, s)^{2}\right)-\psi(t, s) .
$$

After some simple manipulations one arrives at the desired formula (A.11). 


\section{References}

[1] J. Asch, P. Duclos, P. Exner: Stability of driven systems with growing gaps, quantum rings, and Wannier ladders, J. Stat. Phys. 92 (1998) 1053-1070.

[2] D. Bambusi, S. Graffi: Time quasi-periodic unbounded perturbations of Schrödinger operators and KAM methods, Commun. Math. Phys. 219 (2001) 465-480.

[3] J. M. Barbaroux, A. Joye: Expectation values of observables in time-dependent quantum systems, J. Stat. Phys. 90 (1998) 1225-1249.

[4] J. Bellissard: Stability and instability in quantum mechanics, in Trends and Developments in the Eighties, Albeverio Blanchard Eds., (Word Scientific, Singapore, 1985) pp. 1-106.

[5] R. Bhatia, P. Rosenthal: How and why to solve the operator equation $A X-X B=$ $Y$, Bull. London Math. Soc. 29 (1997) 1-21.

[6] L. Bunimovich, H. R. Jauslin, J. L. Lebowitz, A. Pellegrinotti, P. Nielaba: Diffusive energy growth in classical and quantum driven oscillators, J. Stat. Phys. 62 (1991) 793-817.

[7] S. De Bièvre, G. Forni: Transport properties of kicked and quasiperiodic Hamiltonians, J. Stat. Phys. 90 (1998) 1201-1223.

[8] P. M. Bleher, H. R. Jauslin, J. L. Lebowitz: Floquet spectrum for two-level systems in quasi-periodic time dependent fields, J. Stat. Phys. 68 (1992) 271-310.

[9] M. Combescure: The quantum stability problem for time-periodic perturbations of the harmonic oscillator, Ann. Inst. Henri Poincaré 47 (1987) 62-82, Erratum: Ann. Inst. Henri Poincaré 47 (1987) 451-454.

[10] P. Duclos, O. Lev, P. Šťvíček: On the energy growth of some periodically driven quantum systems with shrinking gaps in the spectrum, J. Stat. Phys. (to appear).

[11] P. Duclos, O. Lev, P. Šťovíček, M. Vittot: Weakly regular Floquet Hamiltonians with pure point spectrum, Rev. Math. Phys. 14 (2002) 531-568.

[12] P. Duclos, P. Štovíček: Floquet Hamiltonian with pure point spectrum, Commun. Math. Phys. 177 (1996) 327-247.

[13] V. Enss, K. Veselić: Bound states and propagating states for time-dependent Hamiltonians, Ann. Inst. Henri Poincaré 39 (1983) 159-191.

[14] R. P. Feynman, A. R. Hibbs: Quantum Mechanics and Path Integrals, McGrawHill, New York, 1965. 
[15] G. A. Hagedorn, M. Loss, J. Slawny: Non-stochasticity of time-dependent quadratic Hamiltonians and the spectra of canonical transformations, J. Phys. A: Math. Gen. 19 (1986) 521-531.

[16] J. S. Howland: Scattering theory for Hamiltonians periodic in time, Indiana J. Math. 28 (1979) 471-494.

[17] A. Joye: Upper bounds for the energy expectation in time-dependent quantum mechanics, J. Stat. Phys. 85 (1996) 575-606.

[18] S. G. Krein: Linear Differential Equations in Banach Space, A.M.S., Providence, Rhode Island, 1971.

[19] G. Nenciu: Adiabatic theory: stability of systems with increasing gaps, Ann. Inst. Henri Poincaré 67 (1997) 411-424.

[20] C. R. de Oliveira: Some remarks concerning stability for nonstationary quantum systems, J. Stat. Phys. 78 (1995) 1055-1066.

[21] C. R. de Oliveira, M. S. Simsen: A Floquet operator with pure point spectrum and energy instability, Ann. Henri Poincaré (to appear).

[22] M. Reed, B. Simon: Methods of Modern Mathematical Physics II, Academic Press, New York, 1975.

[23] L. S. Schulman: Techniques and Applications of Path Integration, John Wiley, New York, 1981.

[24] B. Simon: Quantum Mechanics for Hamiltonians Defined as Quadratic Forms, Princeton University Press, Princeton, 1971.

[25] Wei-Min Wang: Pure point spectrum of the Floquet Hamiltonian for the quantum harmonic oscillator under time quasi-periodic perturbations, Commun. Math. Phys (to appear).

[26] K. Yajima: Scattering theory for Schrödinger equations with potential periodic in time, J. Math. Soc. Japan 29 (1977) 729-743. 